ARTICLE

DOI: $10.1038 / s 41467-017-01741-8$

\title{
NOTCH1 is a mechanosensor in adult arteries
}

Julia J. Mack1, Thiago S. Mosqueiro², Brian J. Archer ${ }^{3}$, William M. Jones ${ }^{1}$, Hannah Sunshine ${ }^{4}$, Guido C. Faas ${ }^{5}$, Anais Briot ${ }^{1}$, Raquel L. Aragón ${ }^{6}$, Trent Su${ }^{7}$, Milagros C. Romay ${ }^{1}$, Austin I. McDonald ${ }^{6}$, Cheng-Hsiang Kuo ${ }^{8}$, Carlos O. Lizama9, Timothy F. Lane 7,10,11, Ann C. Zovein (10 9, Yun Fang8, Elizabeth J. Tarling7,11,12, Thomas Q. de Aguiar Vallim,11,12, Mohamad Navab², Alan M. Fogelman², Louis S. Bouchard ${ }^{3,11,13}$ $\&$ M. Luisa Iruela-Arispe $e^{1,11}$

Endothelial cells transduce mechanical forces from blood flow into intracellular signals required for vascular homeostasis. Here we show that endothelial NOTCH1 is responsive to shear stress, and is necessary for the maintenance of junctional integrity, cell elongation, and suppression of proliferation, phenotypes induced by laminar shear stress. NOTCH1 receptor localizes downstream of flow and canonical $\mathrm{NOTCH}$ signaling scales with the magnitude of fluid shear stress. Reduction of NOTCH1 destabilizes cellular junctions and triggers endothelial proliferation. NOTCH1 suppression results in changes in expression of genes involved in the regulation of intracellular calcium and proliferation, and preventing the increase of calcium signaling rescues the cell-cell junctional defects. Furthermore, loss of Notch1 in adult endothelium increases hypercholesterolemia-induced atherosclerosis in the descending aorta. We propose that NOTCH1 is atheroprotective and acts as a mechanosensor in adult arteries, where it integrates responses to laminar shear stress and regulates junctional integrity through modulation of calcium signaling.

\footnotetext{
${ }^{1}$ Department of Molecular, Cell \& Developmental Biology, University of California, Los Angeles, CA 90095, USA. ${ }^{2}$ Institute for Quantitative and Computational Biology, University of California, Los Angeles, CA 90095, USA. ${ }^{3}$ Department of Bioengineering, University of California, Los Angeles, CA 90095, USA. ${ }^{4}$ Interdepartmental Graduate Program in Molecular, Cellular and Integrative Physiology, University of California, Los Angeles, CA 90095, USA. ${ }^{5}$ Department of Neurology, David Geffen School of Medicine, University of California, Los Angeles, CA 90095, USA. ${ }^{6}$ Molecular Biology Interdisciplinary Graduate Program, Molecular Biology Institute, University of California, Los Angeles, CA 90095, USA. ${ }^{7}$ Department of Biological Chemistry, University of California, Los Angeles, CA 90095, USA. ${ }^{8}$ Department of Medicine, University of Chicago, Chicago, IL 60637, USA. ${ }^{9}$ Cardiovascular Research Institute, University of California, San Francisco, CA 94158, USA. ${ }^{10}$ Department of Ob-Gyn, David Geffen School of Medicine, University of California, Los Angeles, CA 90095, USA. ${ }^{11}$ Molecular Biology Institute, University of California, Los Angeles, CA 90095, USA. ${ }^{12}$ Department of Medicine, David Geffen School of Medicine, University of California, Los Angeles, CA 90095, USA. ${ }^{13}$ Department of Chemistry and Biochemistry, University of California, Los Angeles, CA 90095, USA. Correspondence and requests for materials should be addressed to M.L.I-A. (email: arispe@mcdb.ucla.edu)
} 
T he vascular wall is subjected to physical forces resulting from the rhythmic activities of the heart. As the innermost lining of all blood vessels, the endothelium is uniquely responsive to these forces, particularly shear stress, which is transduced by endothelial cells into molecular signals that coordinate homeostatic responses ${ }^{1-4}$. Laminar shear stress induces elongation of endothelial cells ${ }^{5,6}$, suppression of endothelial cell proliferation, redistribution of focal adhesions, reassembly of junctional complexes, and cytoskeletal organization ${ }^{7,8}$. These cellular responses are complex and require both shear stress sensors and a robust cohort of effector molecules that coordinate rapid changes and physiological adaptations.

Importantly, variations in blood flow result in altered hemodynamic forces throughout the vasculature ${ }^{9}$. These hemodynamic forces play an important role in regulating the phenotype and gene expression of endothelial cells in different regions of the arterial wall ${ }^{10-13}$. The descending thoracic aorta is defined by high laminar shear stress and its resulting endothelial gene profile is "atheroprotective" ${ }^{14}$. In contrast, the inner curvature of the aortic arch is characterized by disturbed blood flow with oscillatory shear stress that promotes an "atheroprone" expression profile $^{15-17}$. In this manner, atherosclerosis is known to occur largely in arterial regions exposed to oscillatory shear stress ${ }^{17}$. Because of the clinical impact of these responses, the mechanisms of endothelial mechanotransduction are of great interest.

Mechanosensors act as the initial responders to changes in the mechanical environment ${ }^{18,19}$. Several of these have been identified including integrins, ion channels, G-protein-coupled receptors, and endothelial cell-cell junctional protein ${ }^{20}$. However, the picture of the key contributors involved in flow mechanosensing remains incomplete. Recently, NOTCH1 has been shown to be flow-responsive and involved in modulating the expression of endothelial inflammatory genes ${ }^{21-23}$. Considering that NOTCH1 expression is retained in adult arterie ${ }^{21}$ and activation of this receptor is dependent on physical forces ${ }^{24}$, we investigated the flow-responsive nature of NOTCH1 signaling to determine its biological significance in adult arteries. Our findings indicate that NOTCH1 signaling responds to laminar flow and that this response scales with the magnitude of shear stress. Furthermore, we show that NOTCH1 protein is able to sense laminar flow by rapidly locating to the downstream pole relative to the flow direction. Our results further reveal that NOTCH1 is required to maintain junctional integrity, promote cell elongation in response to flow, and prevent atherosclerosis in the context of hypercholesterolemia. Overall, these findings indicate that NOTCH1 signaling is required in adult arteries to interpret hemodynamic forces and initiate appropriate biological responses required for vascular homeostasis and atheroprotection.

\section{Results}

NOTCH1 signaling is increased by shear stress. Notch signaling is necessary for arterial specification during development $t^{25-28}$. Importantly, immunohistochemistry of mouse aorta revealed that Notch1 protein was abundant in endothelial cells (Fig. 1a) indicating its continuous expression in adult arteries. Additionally, Notch1 activity was robust, as assessed by reporter mice (RBP-Jk: $\mathrm{H} 2 \mathrm{~B}-$ Venus strain $\left.{ }^{29}\right)$. Venus reporter protein was observed in the endothelium of the descending aorta (Fig. 1b) and the carotid artery (Supplementary Fig. 1a), indicating that Notch1 signaling was active in quiescent, non-angiogenic, aortic endothelium.

To determine the effect of shear stress on NOTCH1 transcripts, we transfected human aortic endothelial cells (HAECs) with GFPRBP-Jk reporter constructs and subjected them to static and flow conditions. Fluorescence imaging revealed a two-fold increase in GFP signal in cultures under laminar flow compared to static conditions (Fig. 1c); this correlated with nuclear presence of NOTCH1 protein (Supplementary Fig. 1b). Transcripts were also increased by $12 \mathrm{~h}$ after exposure to flow (Fig. 1d). By $24 \mathrm{~h}$, transcript levels were 1.6-fold higher than static controls, an increase comparable to PECAM1, a known component of a mechanosensory complex ${ }^{30}$, which is activated two-fold at $48 \mathrm{~h}$ under flow. KLF2 mRNA was used as a positive control to confirm flow conditions (Fig. 1d, right plot). Next, HAECs exposed to flow were subjected to $\gamma$-secretase inhibitor DAPT or vehicle for 6, 12 and $24 \mathrm{~h}$. NOTCH1 expression was increased by nearly two-fold after $12 \mathrm{~h}$ of flow. Importantly this rise in transcript levels was blocked by DAPT (Fig. 1e) suggesting the need for a positive feedback loop mediated by NOTCH1 signaling. We then assessed transcripts for NOTCH1 targets at $12 \mathrm{~h}$ of flow compared to static controls to determine whether flow-dependent NOTCH1 gene activation translated into activation of NOTCH downstream effectors. NOTCH1 targets HES1, NRARP, and FABP4 were increased by flow and blocked by DAPT (Fig. 1f). To determine whether NOTCH1 transcriptional activation correlated with protein levels, we evaluated endothelial cells under static and flow conditions in the presence of DAPT or vehicle for $24 \mathrm{~h}$. Active NOTCH1 protein (NICD) levels increased 1.7-fold under flow compared to static control (Fig. $1 \mathrm{~g}$ and Supplementary Fig. 1c). This activation was blocked by DAPT treatment in both static and flow conditions. These in vitro experiments provide evidence that NOTCH1 is activated under laminar shear stress and that laminar shear stress results in positive feedback on NOTCH signaling.

Nuclear NICD responds to stepwise increases in shear stress. Using a Y-shaped fluidic chamber (Fig. 2a), we generated variable flow speeds on a single monolayer of HAECs. Magnetic resonance imaging (MRI) flow mapping ${ }^{31}$ was used to obtain a vector field plot of flow velocities in the presence of a flow-conditioned monolayer. From the magnitude of the MRI-derived flow speed $\left(\mathrm{m} \mathrm{s}^{-1}\right)$, fluid shear $\left(\right.$ dynes $\left.\mathrm{cm}^{-2}\right)$ values were calculated using an assumption of flow between infinitely wide parallel plates and overlaid on the flow vector field plot as a colormap (Fig. 2b). Focusing on a region of high shear $\left(26\right.$ dynes $\left.\mathrm{cm}^{-2}\right)$, endothelial cells were elongated and aligned in the flow direction (Fig. 2c) as assessed by staining for $\beta$-catenin. Staining for NOTCH1 revealed polarization of the protein as clusters at the downstream pole of the cell (Fig. 2c). In contrast, regions of low shear (10 dynes $\mathrm{cm}$ ${ }^{-2}$ ) showed lack of cell elongation (Fig. 2d). Side-by-side comparisons of high and low shear stress regions revealed a marked increase of NOTCH1 protein in the nuclei of endothelial cells under high laminar flow (Fig. 2e). To determine whether nuclear NOTCH1 levels were responsive to shear stress levels, we measured nine regions of interest (ROIs). We then set a threshold value for NOTCH1 protein and determined the percentage of cells with nuclear NOTCH1 in each ROI (Fig. 2f) uncovering a positive correlation of nuclear translocation of NOTCH1 with increasing shear stress. The relationship was logarithmic, with large increases in NOTCH1 nuclear translocation at initially small increases in shear stress, followed by a response plateau starting at 26 dynes $\mathrm{cm}^{-2}$. The findings implied that NOTCH1 activation was finely tuned with flow and reached a maximal activation (saturation) above a specific flow-induced shear stress level.

Laminar fluid flow polarizes NOTCH1 protein. To distinguish NOTCH1 protein polarization from NOTCH1 signaling, we used distinct antibodies for NOTCH1 intracellular domain (ICD) or extracellular domain (ECD), with the objective of visualizing both total protein and activated (cleaved) protein. Analysis of NOTCH1 protein distribution under flow revealed NOTCH1 

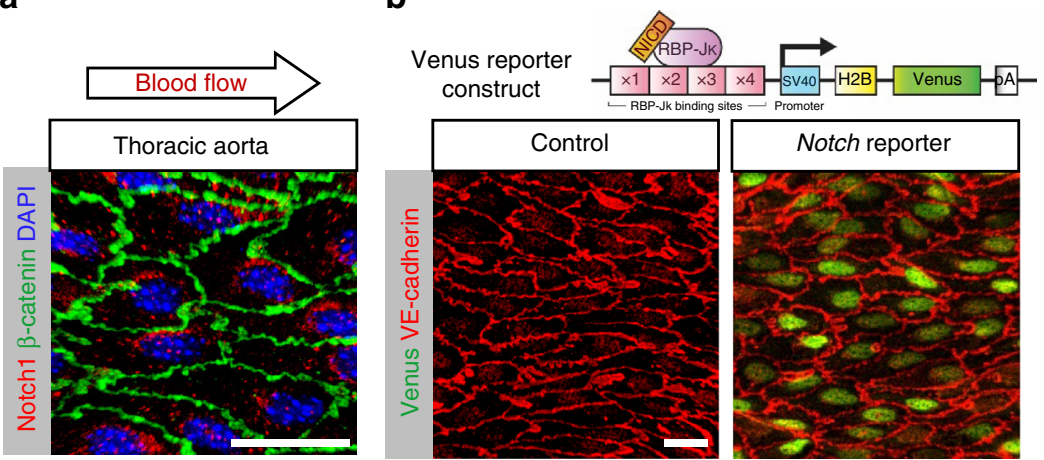

c
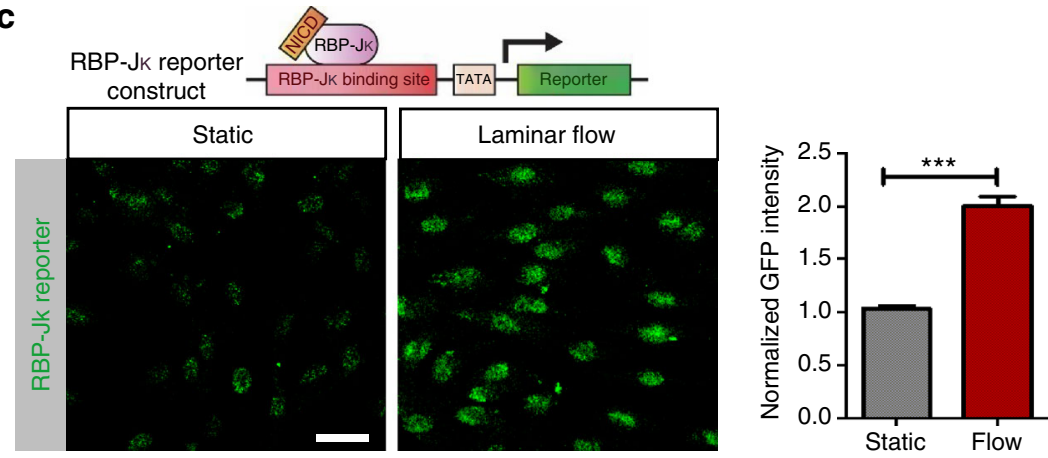

d

Gene expression compared to static control

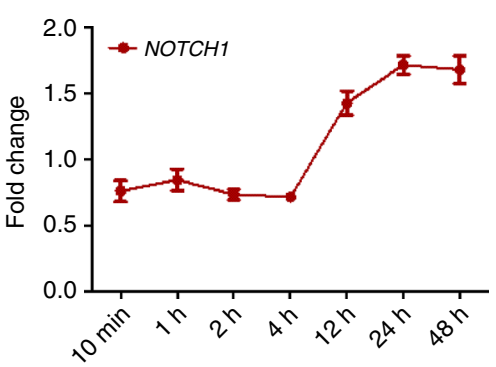

f

NOTCH1 transcript targets compared to static control

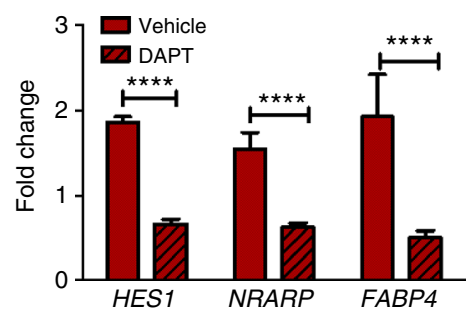

Gene expression compared to static control

g

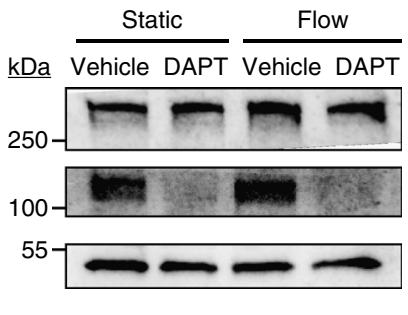

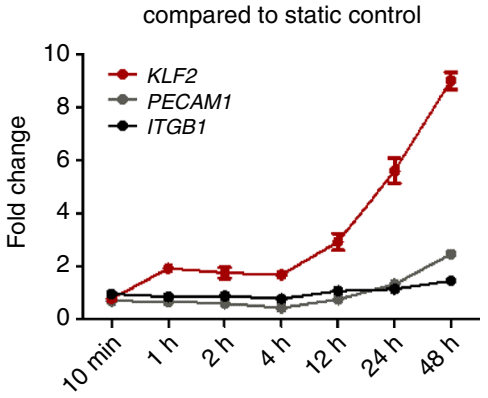

e

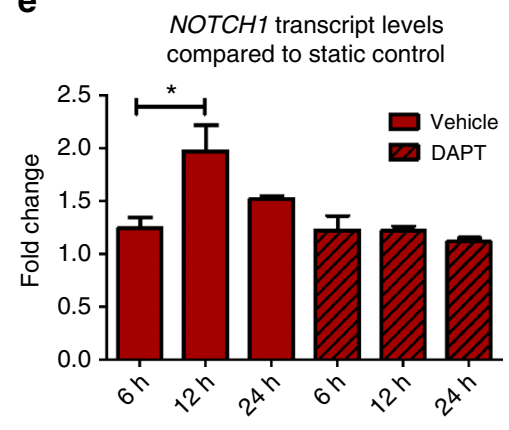

NOTCH1 transcript levels ompared to static control

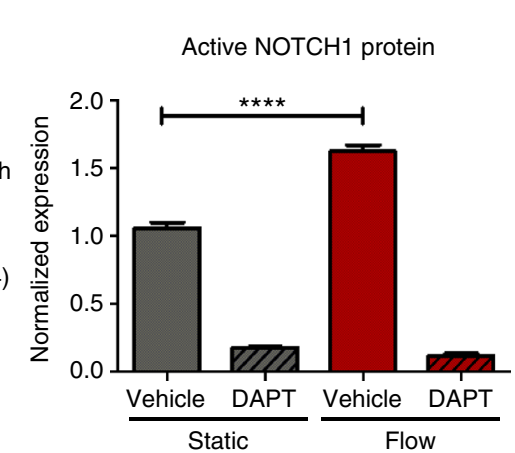

Fig. 1 Notch1 is activated by shear stress in vitro. a En face confocal imaging of wildtype (C57BL/6) adult mouse thoracic endothelium shows Notch1 (red). Staining was done in 20 mice of different strains with identical results, scale bar $=20 \mu \mathrm{m}$. b En face imaging of Venus Notch reporter mouse (RBP-Jk:H2BVenus transgenic) compared to control aorta imaged using identical settings. Note that levels of reporter vary amongst cells indicating distinct degrees of activation in the intima at a given time. Scale bar $=20 \mu \mathrm{m}$. c HAECs transfected with GFP-RBP-Jk reporter showed a two-fold increase in GFP signal intensity under flow (20 dynes $\mathrm{cm}^{-2}$ ) compared to static control at $24 \mathrm{~h}$ (plotted is average GFP intensity \pm SEM of $\sim 120$ cells per condition, from three biological replicates). $T$-test, ${ }^{\star \star \star} P<0.001$. Scale bar $=20 \mu \mathrm{m}$. d Onset of flow (from 10 min to $48 \mathrm{~h}$ ) increases NOTCH1 mRNA after $4 \mathrm{~h}$ for HAECs. Transcript levels of KLF2, PECAM1, and ITGB1 were also profiled over time and compared to static controls. Data shown include mean \pm SEM, $n=4$ biological replicates. e NOTCH1 transcript levels assessed by NanoString chip technology. HAECs were subjected to laminar shear stress for 6,12 , and $24 \mathrm{~h}$ with $\gamma$-secretase inhibitor DAPT or vehicle and compared to vehicle treated static controls. Graph bars represent mean \pm SEM, $n=4$ biological replicates, statistical significance for $6 \mathrm{~h}$ and $12 \mathrm{~h}$ : Wilcoxon rank-sum test, $P=0.0236$. f Flow response of NOTCH1-target genes HES1, NRARP, and FABP4 was determined in HAECs after $12 \mathrm{~h}$ of laminar flow while treated with DAPT or vehicle and compared to vehicle-treated static controls. Graph bars represent mean \pm SEM,$n=5$ biological replicates. $T$-test, ${ }^{\star \star \star} P<0.001$. $\mathbf{g}$ Endothelial monolayers were flow-conditioned for $24 \mathrm{~h}$ in the presence of DAPT or vehicle and protein lysates were analyzed by immunoblot to determine NICD expression levels $\left(n=5\right.$ biological replicates, ${ }^{\star \star \star \star} P<0.0001$ by $t$-test) 
a

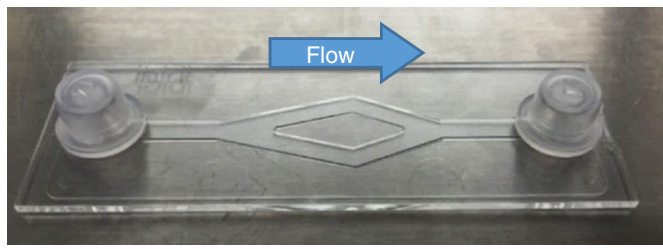

c

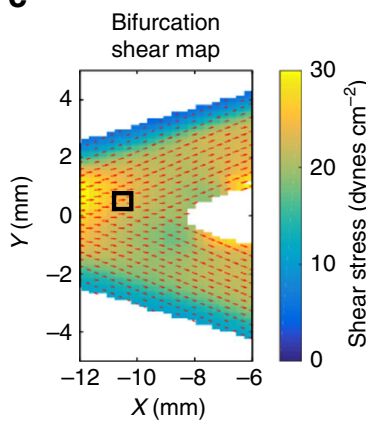

b

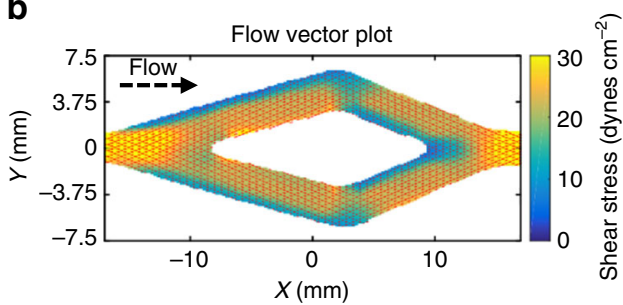

d

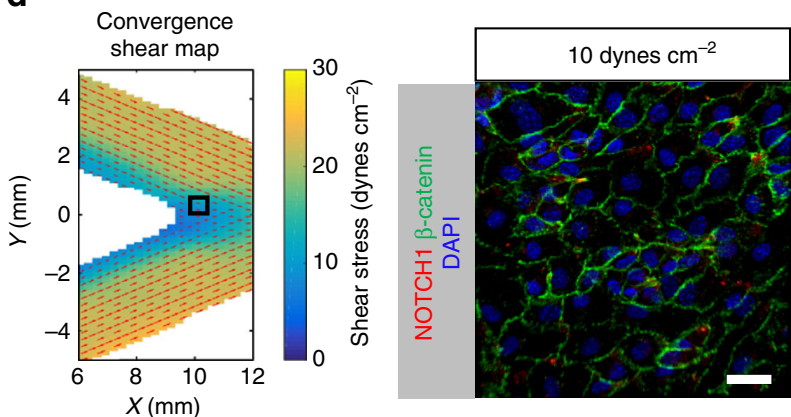

e
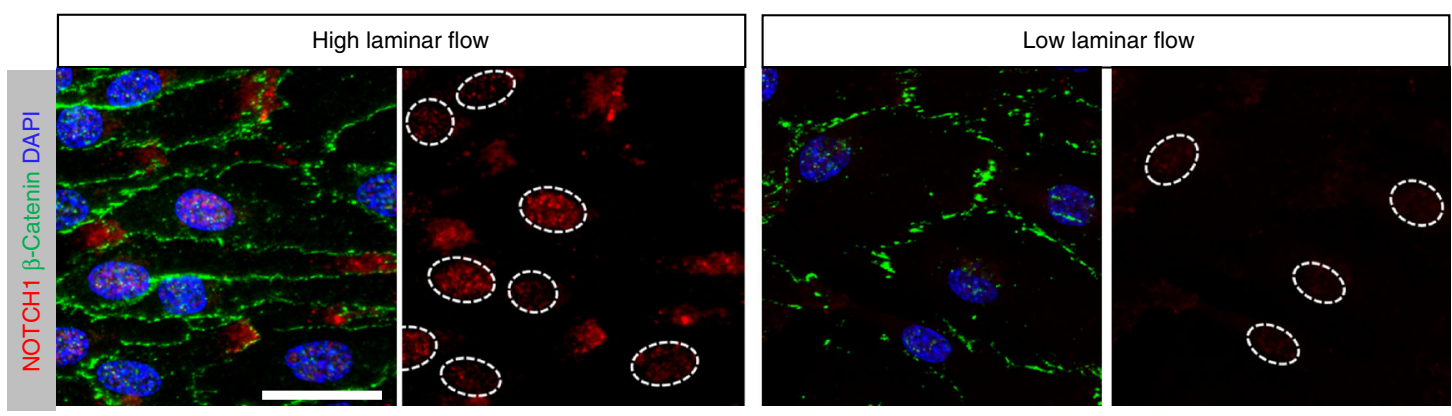

f
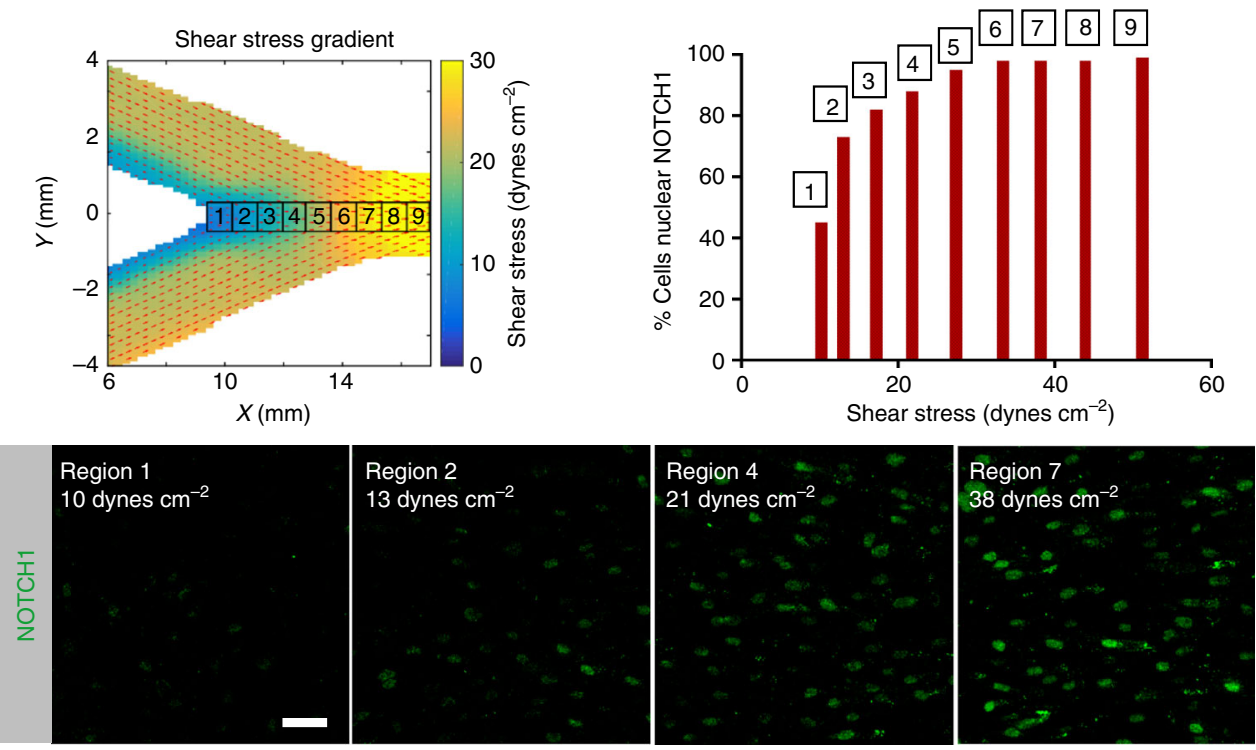

Fig. 2 Shear stress potentiates activation and consequent nuclear translocation of NOTCH1 protein. a Endothelial cells (HAECs) were plated to confluency on a y-slide (ibidi) and subjected to laminar flow ( $9 \mathrm{~mL} \mathrm{~min}^{-1}$ applied). b MRI flow mapping of $\mathrm{y}$-slide in the presence of flow-conditioned HAECs (48 $\mathrm{h}$ ) generated flow vector plots across all regions of the $y$-slide $(n=5)$. c Flow vector plot of high flow speed (26 dynes $\mathrm{cm}^{-2}$ ) region and corresponding $\mathrm{HAEC}$ morphology. Notice cell shape defined by $\beta$-catenin expression at cell-cell borders (green) and polarized NOTCH1 (red) protein. Scale bar $=20 \mu \mathrm{m}$. $\mathbf{d}$ Flow vector plot of low shear stress (10 dynes $\mathrm{cm}^{-2}$ ) region and corresponding HAEC morphology with $\beta$-catenin (green) and NOTCH1 (red). Scale bar $=20 \mu \mathrm{m}$. e Staining for $\mathrm{NOTCH} 1$ (red) in high-flow region compared to low-flow region. Dashed white ovals reveal increase of nuclear NOTCH1 protein under high flow compared to low flow. Scale bar $=20 \mu \mathrm{m}$. f Flow vector plot of the back end of the $y$-slide for nine regions of interest. NOTCH1 (green) nuclear staining was quantified for each of the nine regions and plotted as a function of the measured wall shear stress. For each measurement, $\sim 100$ cells were evaluated using $n=5$ biological replicates, scale bar $=20 \mu \mathrm{m}$ 
localization to cluster at the downstream pole for both the ICD and ECD with some ICD observed in the nucleus (Fig. 3a). In these cells, the ECD was noted only downstream of flow direction. Higher magnification showed ECD at the downstream pole where we also found ICD (Fig. 3b, solid arrowhead). Although we observed the majority of polarized ICD and ECD co-localizing in yellow (Fig. 3b), clusters of cleaved NOTCH1 were also present (Fig. 3b, open arrowhead). These results suggested that both full-length and active NOTCH1 protein were polarized downstream of flow in aligned endothelial cells. To determine whether NOTCH1 protein polarization was flow direction dependent, we reversed the flow direction for $24 \mathrm{~h}$ and observed that NOTCH1 protein re-distributed to the new downstream pole (Fig. 3c). The kinetics of protein re-distribution was evaluated for over 100 individual cells at multiple time points to reveal that re-polarization occurred within $1 \mathrm{~h}$ of flow direction reversal (Supplementary Fig. 2). To confirm whether this flowinduced polarity was biologically relevant or merely an artifact of in vitro culture, we performed a surgical arterial denudation injury $^{32}$ on the mouse thoracic aorta (Fig. 3d schematic) and waited $48 \mathrm{~h}$ post operation to allow for progression of the two migrating endothelial fronts of the wound. Staining for Notch1 protein showed localization downstream of blood flow direction in both migrating fronts, indicating that Notch1 protein polarizes downstream of flow regardless of cell migration direction (Fig. 3d).

NOTCH1 is required for endothelial cell responses to flow. To determine the relevance of NOTCH1 under flow conditions, protein depletion by siRNA knockdown (KD) and Scramble (control) endothelial cells were evaluated under flow (Supplementary Fig. 3a). NOTCH1 protein and transcript levels were assessed over time to validate experimental time points (Supplementary Fig. 3b-d). NOTCH1 KD monolayers displayed gaps between endothelial cells (Fig. $4 \mathrm{a}$ ) with reduction of flow-induced orientation compared to control monolayers (Fig. 4b). We observed a significant decrease in the percent area of $\beta$-catenin coverage, correlating with the presence of gaps (marked in white) (Fig. 4a). Furthermore, discontinuous cell-cell junctions, revealing gaps between cells, were noted in the DAPT treated cultures exposed to flow (Supplementary Fig. 4a, b). We also calculated the orientation angle (degrees) of HAECs with respect to the flow direction $^{33}$. This revealed that while the control monolayers exhibited flow alignment, within $10^{\circ}$ of flow direction, NOTCH1 $\mathrm{KD}$ cells were less aligned, averaging $37^{\circ}$ off of the flow direction, with a much wider distribution of angles (Fig. 4b). To further inspect NOTCH1 KD endothelial cell response to flow, we stained for Golgi complex markers (Supplementary Fig. 4c), which have been shown to polarize either downstream or upstream of the nucleus in response to laminar flow in endothelial cells ${ }^{34}$. For control monolayers, the vast majority $(>80 \%)$ of cells presented with the Golgi located either downstream or upstream of flow direction; however the NOTCH1 KD cells showed a lack of polarization with $<30 \%$ of the cells showing Golgi polarization. Next, we determined the elongation factor of endothelial cells by staining for $\beta$-catenin and measuring the cell length along the flow direction divided by the cell width ${ }^{5}$. Control monolayers displayed an average elongation factor of 4.5 while the NOTCH1 KD monolayers had a reduced elongation factor of 2 (Supplementary Fig. 4d). Once flow aligned, NOTCH signaling was continually required to maintain elongated cell phenotype under flow (Supplementary Fig. 4e). Combined, these data indicate that NOTCH1 is required for endothelial cells to maintain cell-cell junctions, elongated cell morphology, and alignment with flow.
Absence of NOTCH1 promotes endothelial cell proliferation. Evaluation of flow-treated NOTCH1 KD monolayers revealed a two-fold increase in cell density compared to control under flow (Fig. 4c). Cell density was also assessed in cultures subjected to flow and treated with DAPT or vehicle with identical findings (Supplementary Fig. 5a). Interestingly, assessment of CCND1 (Cyclin D1) transcript levels showed suppression under flow exposed to vehicle, but levels were elevated and close to identical between the static and DAPT monolayers exposed to flow (Fig. 4d). We found that endothelial alignment and flow conditioning in the presence of NOTCH1 signaling were not sufficient to prevent the subsequent upregulation of CCND1 once NOTCH1 signaling was inhibited (Fig. 4e). We confirmed the increase in Cyclin D1 protein levels in the absence of NOTCH1 in independent cultures by immunoblot (Supplementary Fig. 5b) and found that $\gamma$-H2A.X (phospho-S139), a marker of DNA double-strand breaks recruited during mitosis ${ }^{35}$, was increased by almost three-fold in flow-conditioned monolayers when NOTCH1 signaling was inhibited (Fig. 4f). Increased $\gamma$-H2A.X protein was also observed in static cultures for NOTCH1 KD cells compared to control (Supplementary Fig. 5c). Further transcriptional profiling of cells cultured under flow with and without NOTCH blockade, indicated that arterial marker EPHRIN B2 was significantly downregulated, but other flow-responsive genes, i.e., KLF2 and KLF4, were unaffected (Fig. 4g). This implied that NOTCH1 signaling was required for maintenance of arterial phenotype and suppression of cell cycle, despite the normal response of other flow-responsive genes indicating that NOTCH1 is necessary for the suppression of cell proliferation.

Deletion of Notch1 in mice reproduced in vitro findings. To further explore biological relevance, we used a transgenic mouse model to enable endothelial-specific inducible deletion of Notch1 in the adult ( $>8$ weeks of age, Notch $1^{\text {ECKO) }}$ (Fig. 5a). Notch1 deletion was confirmed by qPCR (Fig. 5b) and activation of Crerecombinase at the cellular level was validated by tdTomato reporter expression (Fig. 5c). Confocal imaging of the endothelium and staining for endothelial junctional marker Ve-cadherin revealed changes in endothelial cell orientation, as evidenced by instances of cells orientating perpendicular to flow direction at 1 week post Notch1 deletion (Fig. $5 c$; dashed ovals). Measuring cell shape and elongation factor for agematched control littermates vs. Notch $1^{\mathrm{ECKO}}$ mice, we found a statistically significant reduction in endothelial cell elongation factor from 3.5 to 2.3. These findings indicate that Notch 1 signaling was required to maintain flow-induced polarity of endothelial cells. We also found discontinuity in cell-cell junctions in the Notch $1^{\text {ECKO }}$ endothelium, more clearly noted when aortas were stained for fibrinogen ${ }^{36}$ and quantified for fibrinogen deposition (Fig. 5d). At 2 weeks post endothelial Notch1 deletion, we also observed proliferating endothelial cells (white arrow heads) in the descending aorta (Fig. 5e). Quantification of EdU incorporation for Notch 1 ECKO aortic endothelium compared to tamoxifen injected littermate controls (Fig. 5f) indicated a significant enhancement (over two-fold) in endothelial cell proliferation as shown by green fluorescence signal (Fig. 5g). These experiments point to an essential requirement for Notch1 in flow-induced responses associated with cell-cell junctions, endothelial cell polarity, and suppression of proliferation in adult arteries.

Transcriptional changes induced by NOTCH1 reduction. Transcriptome analysis of siNOTCH1 and control HAECs were evaluated to identify differences ${ }^{21}$. A heatmap revealed that reduction of NOTCH1 affected cell cycle, inflammation, and 
a

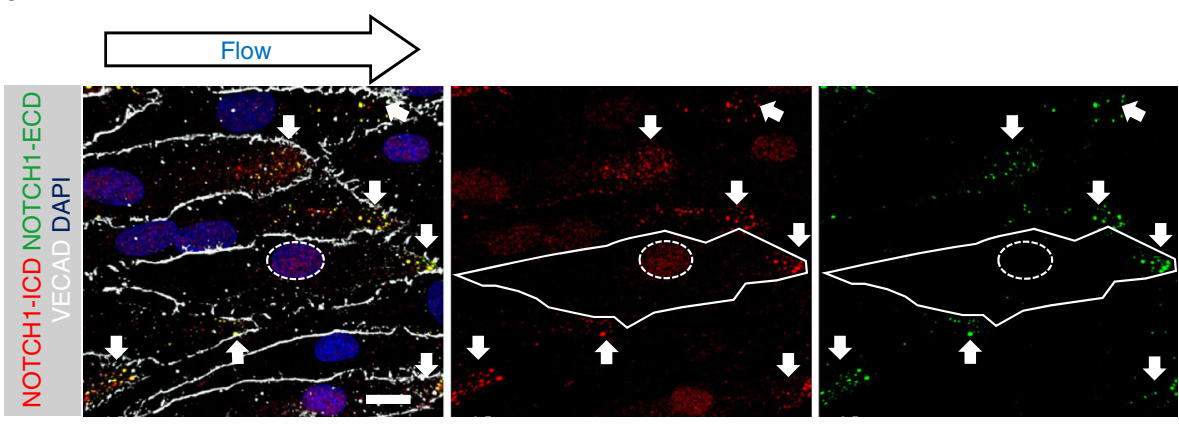

b
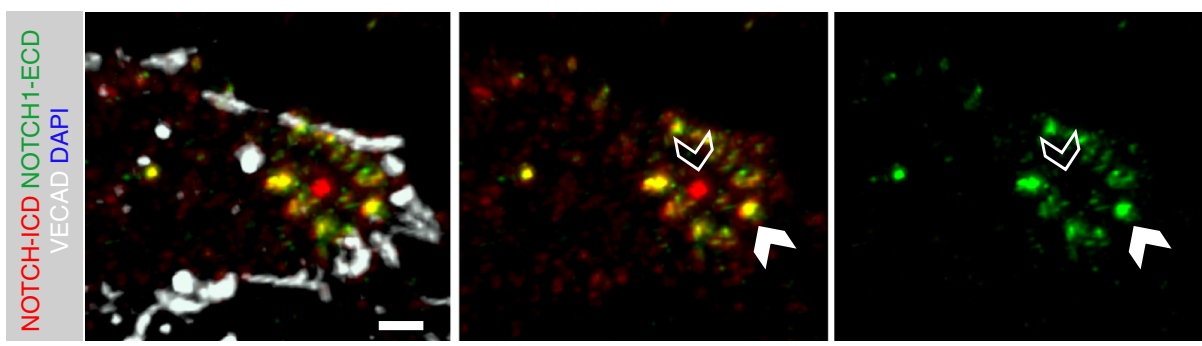

C
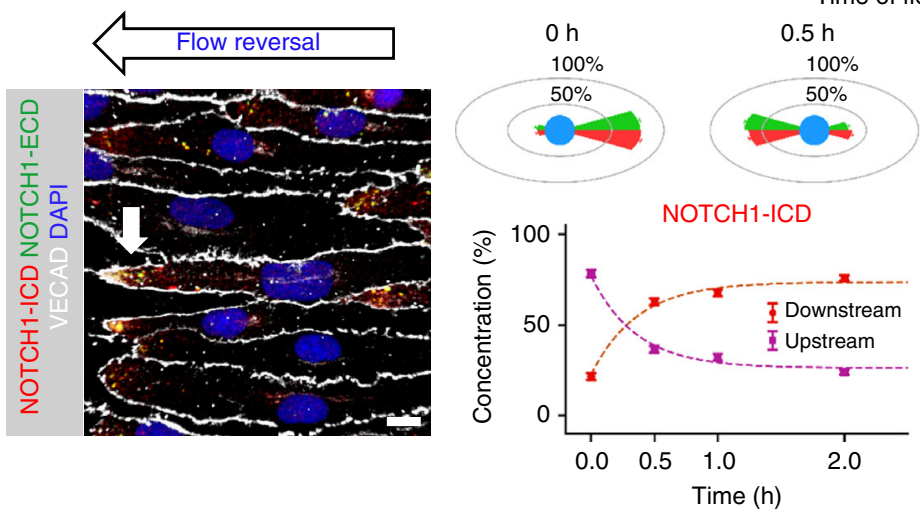

Time of flow reversal $(\mathrm{h})$

d
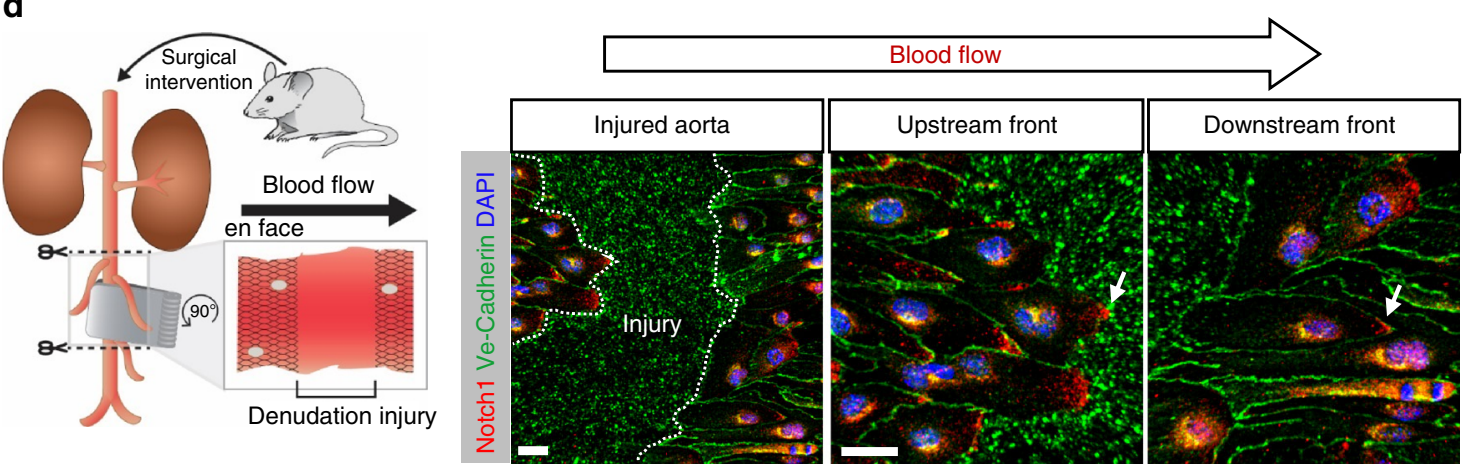

Fig. $3 \mathrm{NOTCH} 1$ is polarized downstream of flow. a Endothelial cells (HAECs) subjected to high laminar flow (20 dynes $\mathrm{cm}^{-2}$ ) display significant cellular elongation, revealed by VECAD (white) and distribution of NOTCH1 intracellular domain (ICD, red) and extracellular domain (ECD, green) at the downstream pole downstream pole, as marked by solid white arrows. Dashed line marks a cell nucleus confirming the nuclear presence of NOTCH1 ICD without ECD. Scale bar $=10 \mu \mathrm{m}$. b Downstream pole of an endothelial cell subjected to high laminar flow showing NOTCH1 ECD (green) and ICD (red). Colocalization of ECD and ICD is indicated by the solid arrowhead and intracellular activated NOTCH1 is marked by the open arrowhead; scale bar $=3 \mu \mathrm{m}$. $\mathbf{c}$ Upon flow-direction reversal, NOTCH1 ICD and ECD protein redistributes to the new downstream pole; scale bar $=10 \mu \mathrm{m}$. Kinetics of the protein redistribution was measured and the typical time for repolarization (dashed lines) was $\sim 30 \mathrm{~min}$ ( $n \geq 116$ individual cells for each time point measured). d Arterial denudation injury of wildtype (C57BL/6) adult mouse to generate injury region (marked by dotted white line) with two migrating endothelial fronts. Note the presence of Notch1 (marked by white arrows) at the downstream pole of the cell regardless of migration direction (at least 40 cells at the wound margins were evaluated in three distinct wounded animals). Scale bars $=20 \mu \mathrm{m}$ 
calcium-dependent pathways (Fig. 6a). Volcano plots of the data visualizes the differentially expressed genes in black with specific cell cycle genes highlighted in red, inflammatory genes highlighted in orange, and genes regulated by intracellular calcium in green (Fig. 6b-d; Supplementary Tables 1-3 lists highlighted genes). NFATs are a family of calcium-dependent transcription factors that are stimulated by a rise in cytoplasmic $\mathrm{Ca}^{2+}$ and the translocation of NFAT proteins to the nucleus requires the phosphatase calcineurin ${ }^{37}$, which is activated by increase of cytosolic $\mathrm{Ca}^{2+}$. Staining for NFAT2 in endothelial monolayers under flow revealed gaps (Fig. 6e, arrows indicating gaps between cells) and nuclear NFAT2 (Fig. 6f, arrows) in monolayers where NOTCH1 signaling was blocked by $\gamma$-secretase inhibitor DAPT. These data pointed to a dysregulation of intracellular $\mathrm{Ca}^{2+}$ in endothelial cells lacking NOTCH1 signaling. a
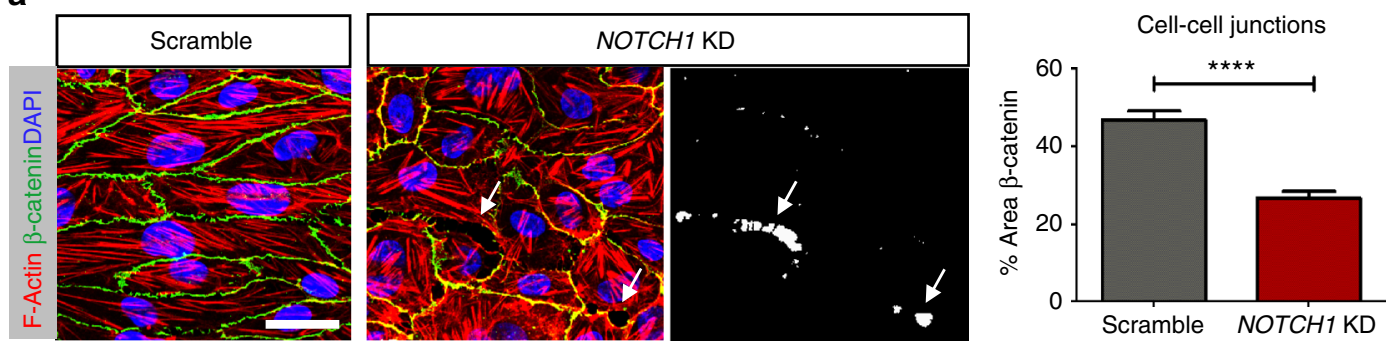

b
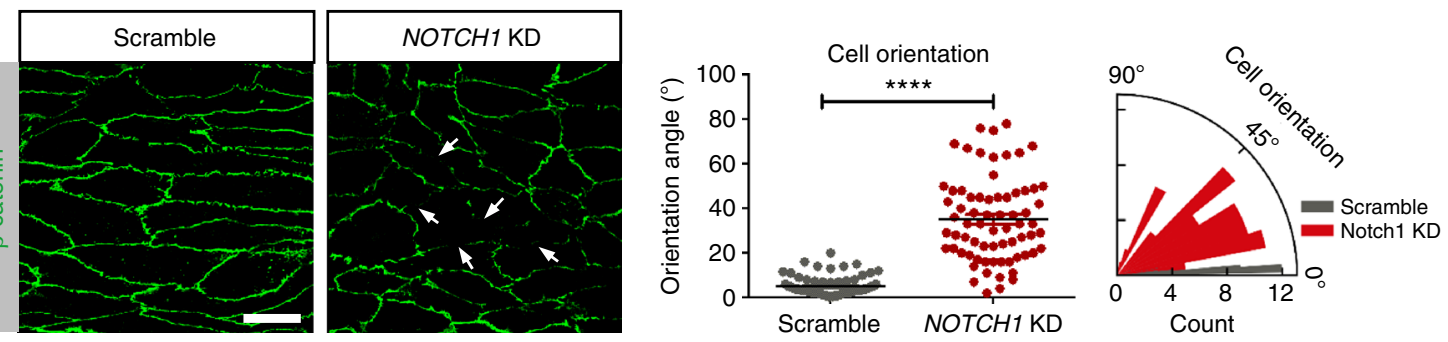

c
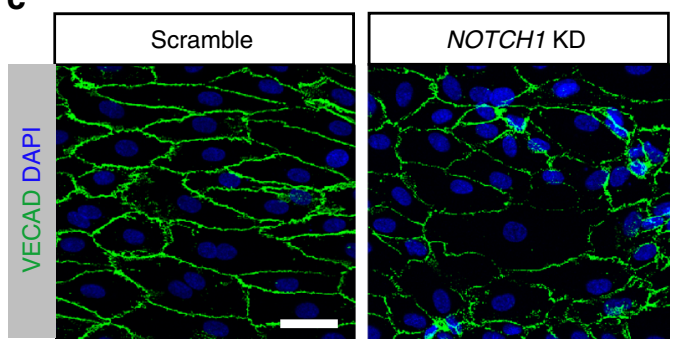

d

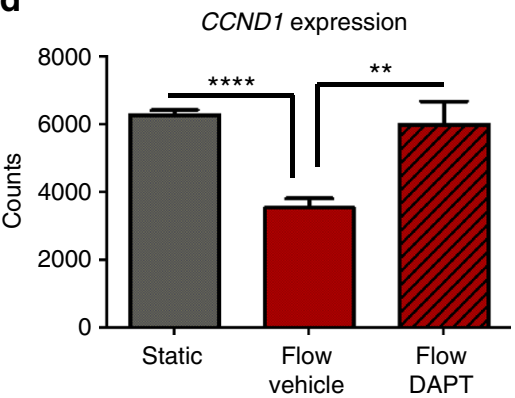

$\mathbf{f}$

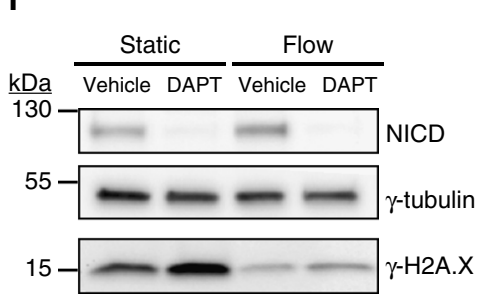

e

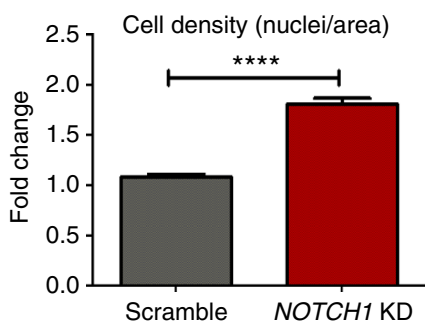

e

CCND1 expression

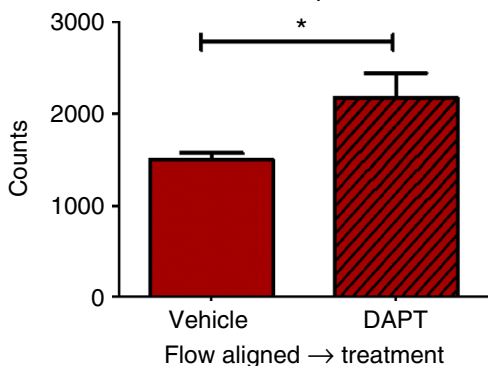

g

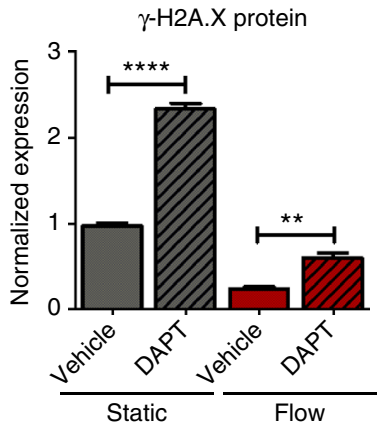


Intracellular $\mathrm{Ca}^{2+}$ is modulated by NOTCH1 under flow. With transcriptome analysis revealing the upregulation of a significant number of intracellular calcium-dependent genes, we measured intracellular $\left[\mathrm{Ca}^{2+}\right]$ changes triggered by the onset of flow, in the presence and absence of NOTCH1 signaling. Confluent monolayers of HAECs loaded with the fluorescent $\mathrm{Ca}^{2+}$ indicator Oregon Green BAPTA-1 were monitored for changes in fluorescence intensity under laminar flow. In each experiment, 81-128 individual cells were measured simultaneously with $1 \mathrm{~s}$ intervals over $10 \mathrm{~min}$ of applied flow (Supplementary Movies 1 and 2). Both groups had comparable resting intracellular $\left[\mathrm{Ca}^{2+}\right]$ levels, 125 and $121 \mathrm{nM}$, respectively. The majority of endothelial cells responded to the onset of flow with one or more temporary increases in $\left[\mathrm{Ca}^{2+}\right]$, i.e., $\mathrm{Ca}^{2+}$ spikes (representative traces in Fig. 7a).

Over the $10 \mathrm{~min}$, the total number of cells that responded to flow was $87 \%$ for Scramble and $94 \%$ for NOTCH1 KD. The average number of spikes in responding cells was increased in NOTCH1 KD (Fig. 7b). Although the spike profiles were similar (Fig. 7c), further analysis indicated that reduction of NOTCH1significantly changed the average characteristics (Supplementary Fig. 6a) of the $\mathrm{Ca}^{2+}$ spikes: duration, amplitude, and area under the spikes. The average duration of the $\mathrm{Ca}^{2+}$ spikes was larger for NOTCH1 KD cells compared to Scramble, $10.7 \pm 4.1$ vs. $8.6 \pm 0.7 \mathrm{~s}$ (Fig. $7 \mathrm{~d}$ ). The average $\mathrm{Ca}^{2+}$ spike amplitudes were larger for NOTCH1 KD than Scramble, $\Delta\left[\mathrm{Ca}^{2+}\right]=7.8 \pm 0.6$ vs. $4.1 \pm 0.3 \mathrm{nM}$ (Fig. $7 \mathrm{e}$ ) and the average area under each spike in NOTCH1 KD $(74 \pm 6 \mathrm{nM})$ was significantly larger for Scramble $(33 \pm 3 \mathrm{nM}$ ) (Fig. $7 \mathrm{f}$ and Supplementary Fig. 6b). From these results, we concluded that a reduction in NOTCH1 leads to increased flow triggered $\mathrm{Ca}^{2+}$ signaling and hypothesized that this increase in $\mathrm{Ca}^{2+}$ signaling underlies the previously observed cell-cell junctional integrity loss under flow. To test this hypothesis, we flow-treated endothelial monolayers cultured in media with high $(3 \mathrm{mM})$ $\left[\mathrm{Ca}^{2+}\right]$ to increase $\mathrm{Ca}^{2+}$ signaling for $24 \mathrm{~h}$ and compared these with monolayers cultured under flow in physiological $(1.5 \mathrm{mM})$ $\left[\mathrm{Ca}^{2+}\right]$. Monolayers cultured under physiological conditions had continuous cell-cell junctions, whereas those treated with high $\left[\mathrm{Ca}^{2+}\right]$ displayed gaps between cells (Fig. $7 \mathrm{~g}$ ). Conversely, we set out to determine whether reducing intracellular $\mathrm{Ca}^{2+}$ signaling under flow would rescue the cell-cell junctions in the absence of NOTCH1 signaling. For this, we loaded the cell permeable $\mathrm{Ca}^{2+}$ chelator BAPTA-AM (BAPTA is trapped inside the cell after cleavage) or vehicle control into flow-conditioned HAECs. After reagent loading, monolayers were subjected to flow in the presence of DAPT to block NOTCH1 signaling and we quantified the integrity of cell-cell junctions by measuring the area uncovered (Fig. 7h). In monolayers with BAPTA-loaded cells, the area uncovered was significantly reduced when compared to monolayers that were not loaded with BAPTA $(0.56 \pm 0.07 \%$ vs $2.65 \pm 0.36 \%$, Fig. $7 \mathrm{~h}$ ). Control monolayers were evaluated to eliminate the possibility of secondary effects due to vehicle or BAPTA-AM loading (Supplementary Fig. 6c). In the absence of NOTCH1 signaling, BAPTA-loaded monolayers significantly retained cell-cell junctions to a greater degree than vehicletreated monolayers (Fig. 7h). This rescue of the NOTCH1 suppression phenotype further indicates that intracellular $\mathrm{Ca}^{2+}$ dynamics are modulated by NOTCH1 under flow. Taken together, these findings strongly support our hypothesis that reduced NOTCH1 signaling leads to destabilization of cell-cell junctional integrity through increased $\mathrm{Ca}^{2+}$ signaling under flow.

Loss of endothelial Notch1 in vivo results in inflammation. With transcriptome analysis of siNOTCH1 HAECs indicating an enhancement of genes associated with inflammation (Fig. 6a, c) and increase in CD68 expression in the tunica intima of Notch 1 ECKO adult mice (Fig. 8b), we further evaluated Notch1ECKO aortas at 8 weeks post deletion (Fig. 8a) to assess the longterm biological consequences of Notch1 deletion. Imaging of the descending aorta of Notch $1^{\text {ECKO }}$ mice 8 weeks post deletion confirmed the presence of $\mathrm{CD} 45^{+}$inflammatory cells embedded within the tdTomato $^{+}$endothelial layer (Fig. 8c). CD $45^{+}$ inflammatory cells were frequently found between endothelial cells in the Notch $1^{\text {ECKO }}$ descending aorta, but not in controls (Fig. 8d). The presence of these inflammatory cells was manifested in all descending aortae of Notch $1^{\text {ECKO }}$ animals evaluated $(n=10)$. Interestingly, complete blood count (CBC) analysis did not show differences in circulating monocytes, lymphocytes, or neutrophils for Notch $1^{\text {ECKO }}$ animals compared to controls at any time point evaluated (Supplementary Fig. 7a, b). Instead, we found that the $\mathrm{CD} 45^{+}$inflammatory cells potentially proliferated in situ, as evidenced by EdU incorporation (white open arrow heads in Fig. 8e). To determine the topographical location of the inflammatory cells within the aortic wall, we generated a $3 \mathrm{D}$ surface render of the confocal Z-stack images. $\mathrm{CD} 45^{+}$cells were found both at the endothelial surface and in the tunica intima (Fig. 8f and Supplementary Fig. 7c, d). Histological sections of aorta and livers of Notch $1^{\text {ECKO }}$ animals showed an influx of inflammatory cells in close proximity to the vessel lumen (Supplementary Fig. 7e, f). These data point to the requirement of endothelial Notch1 to limit inflammation.

Loss of endothelial Notch1 augments atherosclerotic plaques. To investigate the role of endothelial Notch1 in a pathophysiological setting, we utilized an atherosclerosis mouse model (Fig. 9a) in which hypercholesterolemia was induced via PCSK9-

\footnotetext{
Fig. $4 \mathrm{NOTCH} 1$ is required for maintenance of endothelial cell-cell junctions, cell alignment, and suppression of proliferation under flow. Endothelial cells (HAECs) were treated with siRNA for NOTCH1 or Scramble and flow-conditioned for $48 \mathrm{~h}\left(20\right.$ dynes $\left.\mathrm{cm}^{-2}\right)$. a F-Actin and $\beta$-catenin revealed gaps between cells as indicated with arrows and in white on inverted image; scale bar $=20 \mu \mathrm{m}$. Cell-cell junctions were quantified by percent area $\beta$-catenin coverage, shown is mean \pm SEM from five distinct cultures obtained from five biological replicates. T-test, ${ }^{\star \star \star \star} P<0.0001$. $\mathbf{b} \beta$-Catenin staining to reveal cell shape and determine cell orientation angle (positive value of degrees) with respect to the flow direction. At least 70 cells were evaluated per condition across three biological replicates; scale bar $=20 \mu \mathrm{m}$. $T$-test ${ }^{\star \star \star \star} P<0.0001$. c Increased cell density for NOTCH1 KD compared to control by quantification of nuclei counts; scale bar $=20 \mu \mathrm{m}$. Graphs show data obtained from four biological replicates, $>100$ cells per replicate, and expressed as mean \pm SEM. $T$-test ${ }^{\star \star \star \star} P<0.0001$. d CCND1 transcript was analyzed in HAECs cultured statically or flow-treated with $50 \mu \mathrm{M}$ DAPT or volume equivalent DMSO for $24 \mathrm{~h}$ by NanoString nCounter Gene Expression. Graph bars represent mean \pm SEM, $n=5$. T-test ${ }^{\star \star} P<0.01,{ }^{\star \star \star \star} P<0.0001$. e CCND1 transcript is increased after blocking NOTCH1 signaling for HAEC monolayers that were flow-aligned for $48 \mathrm{~h}$ and subsequently treated with DAPT or vehicle under flow for an additional $12 \mathrm{~h}$. Graph bars represent mean $\pm \mathrm{SEM}, n=5$. T-test ${ }^{\star} P<0.05$. f Monolayers were subjected to flow for $24 \mathrm{~h}$ with $50 \mu \mathrm{M}$ DAPT or vehicle and protein lysates analyzed by immunoblot to quantify expression of $\gamma$-H2A.X to show a 2.7-fold increase in $\gamma$-H2A.X expression for DAPT treated cultures $\left(n=4, T\right.$-test $\left.{ }^{\star \star} P<0.01\right)$. $g$ NanoString $n$ Counter Gene Expression for EFNB2, KLF2, and KLF4 transcripts for HAECs cultured under flow for $24 \mathrm{~h}$ in the presence of $50 \mu \mathrm{M}$ DAPT or DMSO revealed reduction in transcripts for EFNB2, but no statistically significant change for KLF2 or KLF4. Graph bars represent mean \pm SEM, $n=5$. $T$-test ${ }^{\star \star \star \star} P<0.0001$
} 
$\mathrm{AAV}^{38}$. In brief, 2 weeks post tamoxifen, control and Notch $1^{\mathrm{ECKO}}$ animals were injected with PCSK9-AAV or control AAV and subjected to a high-fat diet for 3 months. Throughout the study and every 2 weeks, animal body weights were measured and blood was drawn via retro-orbital bleed to assess circulating lipid levels. At the time of termination, liver protein lysates were used to determine LDLR protein by immunoblot (Fig. 9b). A significant increase in total cholesterol was evident in animals injected with PCSK9-AAV, both control and Notch ${ }^{\text {ECKO }}$ animals (Fig. 9c). No difference in body weight was found between control and Notch $1^{\text {ECKO }}$ animals at 4 weeks post tamoxifen (Supplementary Fig. 8a). Analysis of animals injected with control AAV over the 3 months of high-fat diet, exhibited a slight trend toward increased body weight for Notch $1^{\mathrm{ECKO}}$ animals at time of harvest (Supplementary Fig. 8b). Animals injected with PCSK9 showed the sustained increase in total cholesterol (Fig. 9c

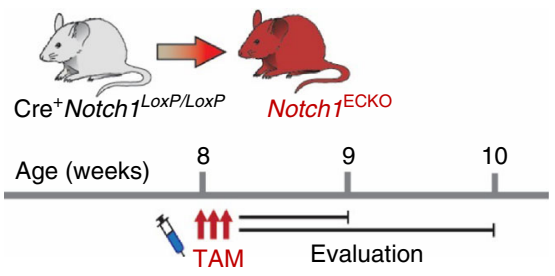

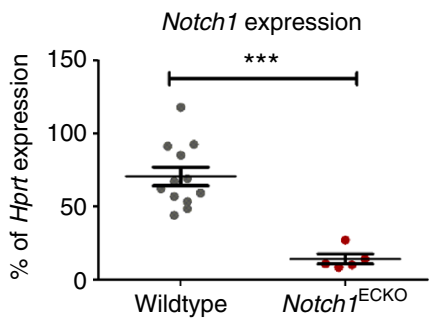
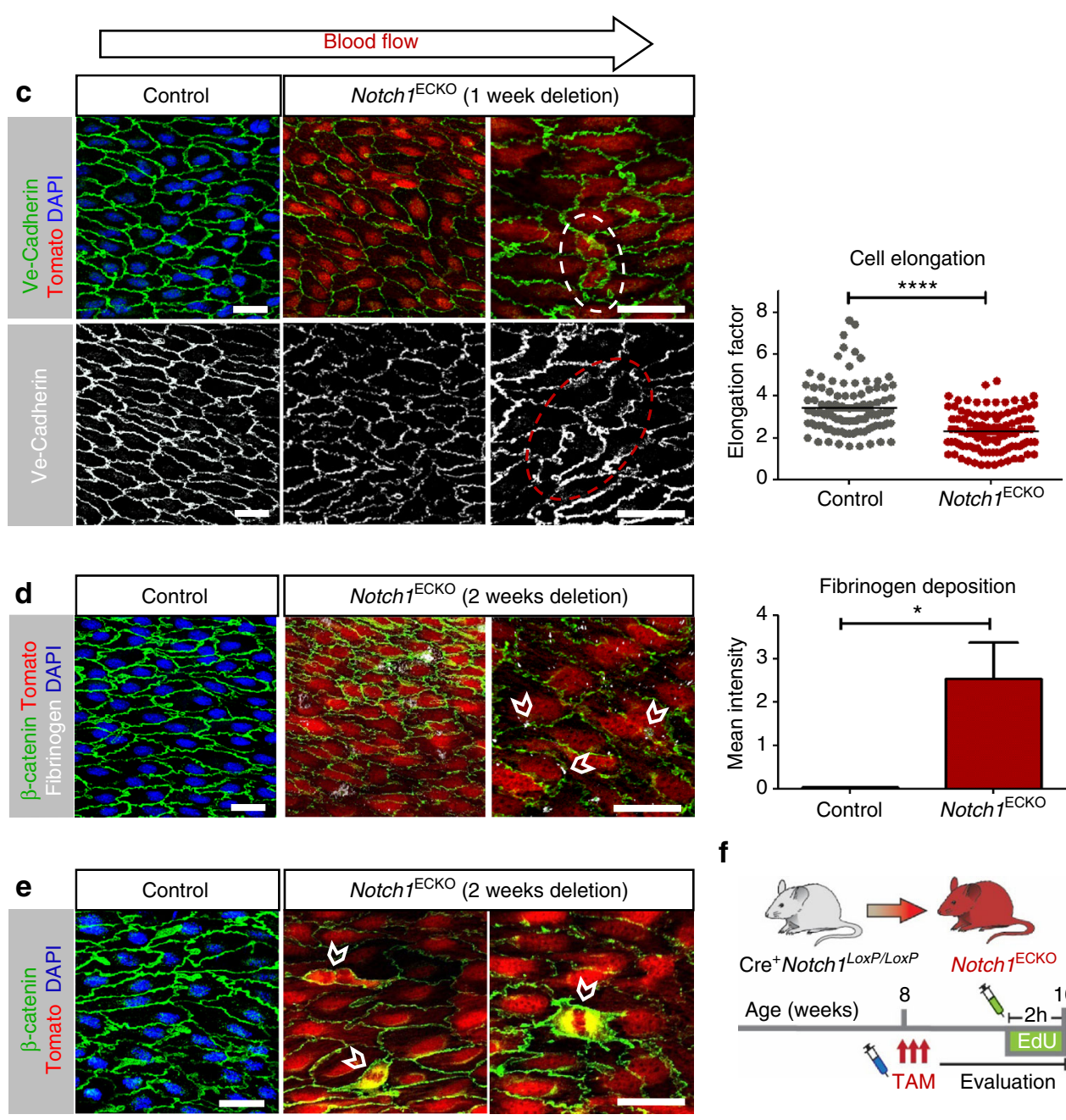

f
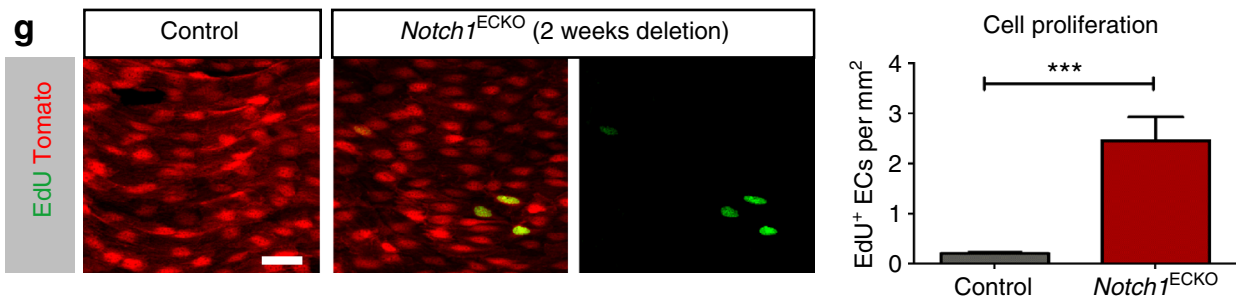
and Supplementary Fig. 8c), but no significant difference in body weight (Supplementary Fig. 8d). Atherosclerotic lesions were observed in all animals injected with PCSK9, however no lesions were found in animals injected with control AAV (Fig. 9d showing four aortas from each group). Sudan IV staining was applied to aortas (Fig. 9e) to quantify plaque burden as a percentage of aorta area, defining the upper region as the Arch and the lower region as the Descending Aorta (marked in dashed boxes in Fig. 9e). Quantification of the plaque area revealed a significant increase in plaque burden for the whole aorta and the descending aorta, but not the arch, for $\operatorname{Notch}_{1}{ }^{\mathrm{ECKO}}$ animals (Fig. 9f).

To further understand the differential outcomes in aortic plaque burden in the descending aorta vs. arch, we probed Notch1 expression in different regions of the aorta (regions marked in Fig. 9g), representing two extreme (low and high) regions of time-averaged shear stress. We found higher frequency of endothelial cells with nuclear Notch1 staining in the descending aorta compared to the lower arch (arrowheads in Fig. 9g). Notch1 activity in the two regions was further assessed using the Notch reporter mouse and mean fluorescence intensity showed a 1.6-fold increase in the descending aorta compared to the lower arch, indicating that Notch activity was amplified in regions of higher shear stress (Fig. 9h). These findings were corroborated biochemically using endothelial-enriched protein lysates isolated from bovine aortas (Supplementary Fig. 8e). Expression levels of Notch1 intracellular domain (NICD) and Klf2 were quantified using Ve-cadherin as a loading control ${ }^{39}$, and we found that NICD levels followed the same trend as Klf2, a recognized indicator of shear stress ${ }^{40,41}$ with a two-fold enhancement in the endothelium of the descending aorta (Fig. 9i; original gels in Supplementary Fig. 9). Comparing lower arch to upper arch revealed a similar trend in Notchl activity, with more NICD in the upper arch where shear stress magnitude is higher (Supplementary Fig. 8f). To probe for the response of NOTCH1 signaling under different types of flow, we compared endothelial monolayers cultured statically or under atheroprotective flow under disturbed flow. NOTCH1 gene expression was enhanced under atheroprotective flow, but not under disturbed flow (Supplementary Fig. 8g). Additionally, the NOTCH1 target HES1 was upregulated under atheroprotective, but not under disturbed flow, following the same trend as KLF2. Investigation of inflammatory gene expression changes under atheroprotective flow revealed an increase in ICAM1, IL 8 and CXCL2 in NOTCH1 $\mathrm{KD}$ monolayers (Supplementary Fig. 8h). Interestingly, the suppression of NOTCH1 did not alter ICAM1, IL8, or CXCL2 gene expression under disturbed flow where average shear stress is very low (Supplementary Fig. 8i). Combined, these data provide evidence that endothelial NOTCH1 signaling correlates positively with the level of shear stress in the aorta in vivo and that the presence of Notch1 in the descending aorta protects the endothelium from atherosclerotic plaque build-up in the advent of hypercholesterolemia.

\section{Discussion}

Responses of endothelial cells to laminar flow include alignment, suppression of proliferation, and acquisition of an antiinflammatory profile $e^{42,43}$. While these phenotypes are recognized landmarks of cells experiencing laminar flow, the mechanisms involved in promoting these effects remained unclear. Here we demonstrate that NOTCH1 signaling is required for all three phenotypes. Reduction of NOTCH1 in vitro or genetic inactivation in vivo results in destabilization of cell-cell junctions and continuous endothelial proliferation irrespective of the presence of flow. Intriguingly, classical markers of flow, like KLF2, appear unaffected by deletion of NOTCH1. The fact that KLF2 is upregulated by flow in the absence of NOTCH1, supports the concept that KLF2 alone is not sufficient to regulate flow responses associated with junctional stability and cell proliferation. For those actions, NOTCH1 is a critical mediator.

During angiogenesis, Notch1 signaling is essential for tip-stalk specification within vascular sprouts ${ }^{44}$ and to facilitate junctional rearrangements ${ }^{45}$. Later, Notch1 is required for arterial fate $^{26,27,46}$, nonetheless the relevance of Notch1 in adult arterial endothelium is unclear. In vitro, NOTCH1 signaling has been reported to increase with shear stress 22,23 , yet activation of Notch1 by flow in vivo has been disputable with findings supporting a direct effect ${ }^{23}$ and others indicating that Notch1 is suppressed by shear stress ${ }^{47,48}$. A critical aspect that might explain these discrepancies relates to the type of flow, endothelial cell type used (lymphatic vs. blood ${ }^{47}$ ), levels of shear stress, and stage in development. Here we demonstrate that only laminar flow increases NOTCH1. Furthermore, we also show that NOTCH1 responds exquisitely to the level of shear stress. In fact, using flow velocity gradients, we demonstrated a highly sensitive direct positive relationship between shear stress and NOTCH1 signaling in real time. Our findings also revealed that NOTCH1 protein is polarized downstream of flow direction regardless of cellular polarity, as indicated by the direction of cell migration in arterial wounds. By changing flow direction in vitro, we further uncovered that this relationship is dynamic and physiologically tuned to its environment with rapid relocation of the protein. Combined, our findings indicate that NOTCH1 pathway is able to sense and respond to physical flow forces. Importantly, NOTCH1 activation requires forces on the order of

Fig. 5 Deletion of Notch1 results in regression of cell alignment, compromised cell-cell junctions, and enhanced proliferation. a Schematic to illustrate time line for in vivo model of inducible deletion of endothelial Notch1 (Notch ${ }^{E C K O}$ ). Cre ${ }^{+}$Notch $7^{\text {loxP/loxP }}$ mice after 8 weeks of age received three intraperitoneal injections of tamoxifen as did age-matched control mice at specified time points. $\mathbf{b}$ Notch1 transcript levels from descending aortae of adult WT mice and Notch $7^{\mathrm{ECKO}}$ mice 2 weeks post tamoxifen measured by qPCR analysis ( $n=12 \mathrm{WT}$ and $n=5$ Notch $7^{\mathrm{ECKO}}$ ). T-test ${ }^{\star \star \star \star} P<0.0001$. c En face confocal imaging of the endothelium of the descending aorta for Notch $7^{\mathrm{ECKO}}, 1$ week post deletion, and control mice (Cre ${ }^{-}$Notch $1^{\text {loxP/loxP}}$ ); all mice were injected with tamoxifen. Ve-Cadherin revealed instances of endothelial cells oriented perpendicular to blood flow direction (highlighted by dashed ovals). Quantification of cell orientation, measured by elongation factor (cell length along flow direction divided by cell width) for Notch $7^{\mathrm{ECKO}} \mathrm{vs}$. control (Cre ${ }^{-}$Notch $7^{\text {loxP/loxP }}$ ). All mice were injected with tamoxifen and evaluated 1 week later (100 cells were measured from 5 mice for each genotype, $T$-test $\star \star \star \star ~ P<0.0001$ ). $\mathbf{d}$ En face imaging of descending aortas 2 weeks post tamoxifen revealed fibrinogen deposited between endothelial cells (open arrow heads) in Notch 7 ECKO animals, but not in age-matched tamoxifen-injected controls. Determination of fibrinogen was obtained by mean fluorescence intensity in areas of $500 \mu m^{2}$ from five mice of each genotype. Graph bars represent mean \pm SEM. $T$-test ${ }^{\star} P<0.05$. e Proliferating endothelial cells, as evidenced by the mitotic figures (solid arrow heads), in Notch $1^{\mathrm{ECKO}}$ aortas but not in control (Cre ${ }^{-}$Notch ${ }^{\text {loxP/loxP }}$ ). f Experimental design to quantify endothelial proliferation by EdU incorporation in the descending aortae of control and Notch $7^{\mathrm{ECKO}}$ animals at 2 weeks post tamoxifen. $\mathbf{g}$ Representative en face images and quantification of EdU-positive endothelial cells per unit area of aorta $\left(\mathrm{EdU}^{+}\right.$endothelial cells per $\left.\mathrm{mm}^{2}\right)$. Notch $7^{\mathrm{ECKO}}$ and control (Cre ${ }^{+}$tdTomato reporter). EdU incorporation is visualized in green. Graph bars represent mean of $\sim 2 \mathrm{~cm}^{2}$ segments of the aorta evaluated from six mice per genotype \pm SEM. $T$-test ${ }^{\star \star \star} P<0.001$. Scale bars $=20 \mu \mathrm{m}$ 
a

\section{NOTCH1 $\quad$ SiNOTCH1/ targets}
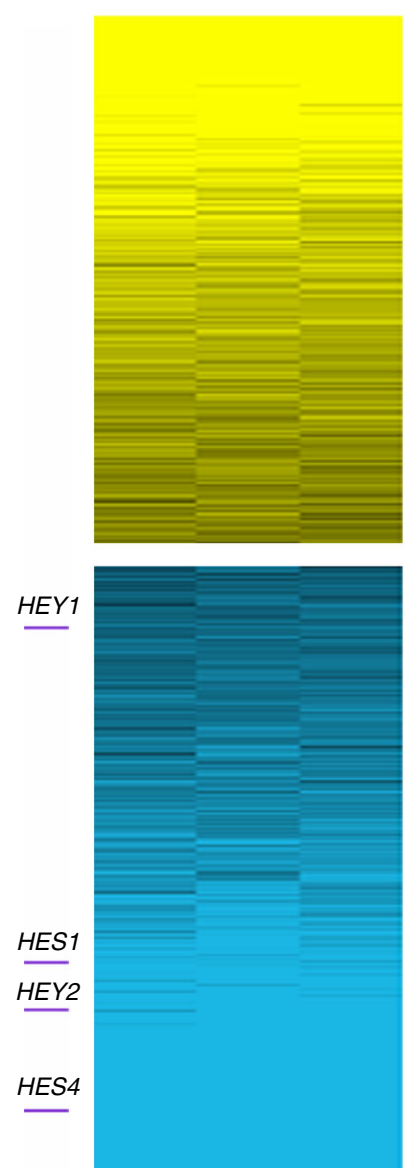

Cell cycle Inflammation Calcium b

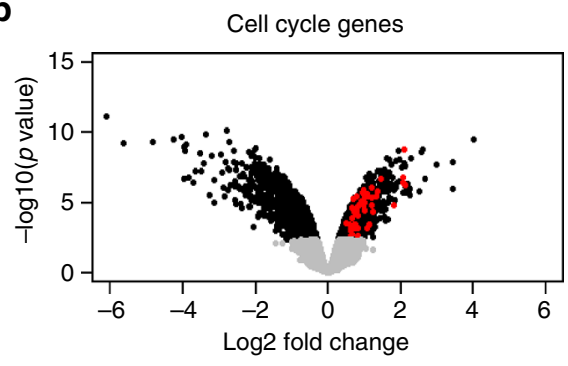

C

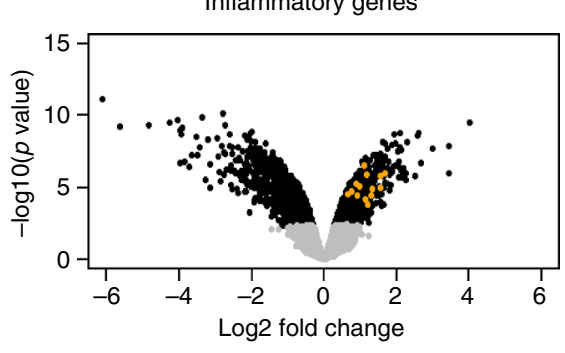

d

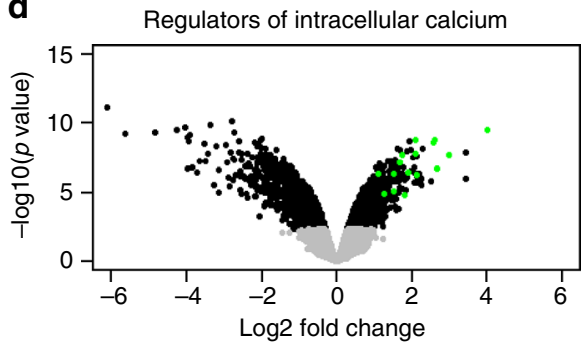

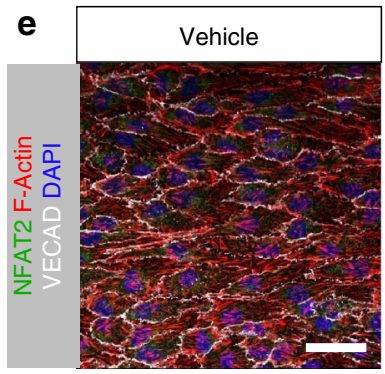
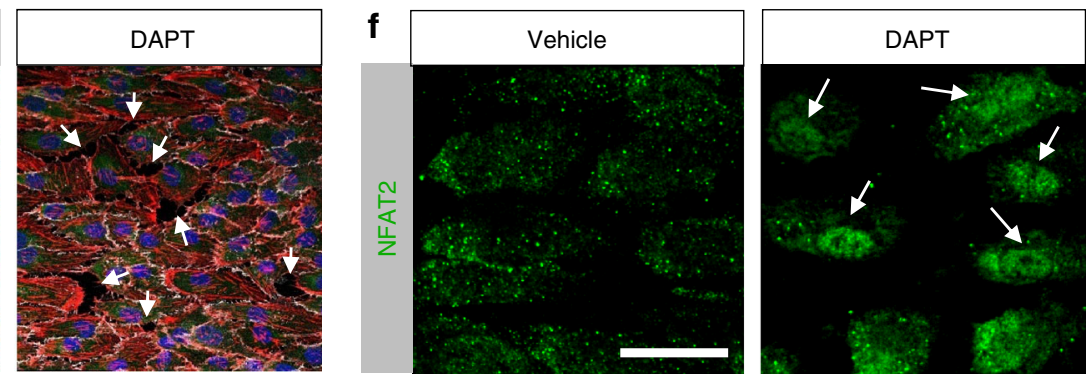

Fig. 6 Transcriptome of siNOTCH1 indicates changes in cell cycle, inflammatory genes, and regulators of calcium. a Cultures of HAECs treated with siNOTCH1 and siControl were analyzed for transcriptional changes by microarray. Heatmap to display differentially expressed genes ( $\geq 0.5$ (log2) upregulated or $\leq-0.5(\log 2)$ downregulated with $P<0.001)$ marking $\mathrm{NOTCH} 1$ target genes and upregulated cell cycle, inflammatory genes and regulators of intracellular calcium. (b-d) Volcano plot visualization of the differentially expressed genes between siNOTCH1 and siControl. Colors indicate as follows: gray-no differential expression, black-genes whose fold change is either $\leq 0.50$ or $\geq 1.5$, red-genes known to contribute to the regulation of cell cycle, orange-genes involved in inflammation, and green-genes known to be regulators of intracellular calcium. (e, $\mathbf{f}$ ) Confocal images of HAECs exposed to flow for $48 \mathrm{~h}$ in the presence of $\gamma$-secretase inhibitor DAPT or vehicle and stained for F-actin, VECAD, and NFAT2. Note the presence of nuclear NFAT2 and gaps between cells only in DAPT-treated monolayers (marked by white arrows). Scale bars $=20 \mu \mathrm{m}$

$12 \mathrm{pN}^{24}$, and therefore constant alterations in cellular tension are likely important contributors to its activation.

As to proliferative responses, it has been reported that NOTCH1 is activated by cell-cell contact under static conditions to promote cell cycle arrest ${ }^{49,50}$. Our data are fully consistent with those in vitro findings and further support the notion that utilization of the NOTCH1 pathway by flow activation is a mechanism by which cell cycle can be titrated and vascular homeostasis preserved. In the absence of NOTCH1 this suppression is muted, regardless of the presence of flow, as demonstrated here by EdU incorporation in the aortic endothelium of Notch $1^{\text {ECKO }}$ mice and presence of mitotic figures. The profile associated with proliferation is robust and suggests that Notch1 suppresses a default state that can actively promote cell cycle entry. Genes found to be transcriptionally increased in the absence of NOTCH1 include cyclins and cdks and several regulators of cell cycle (Supplementary Table 1). This is a vigorous program that clearly shows a commitment to cell division. We also found that transcripts for EFNB2, a marker of arterial identity, are reduced when NOTCH1 is suppressed. The data 
a
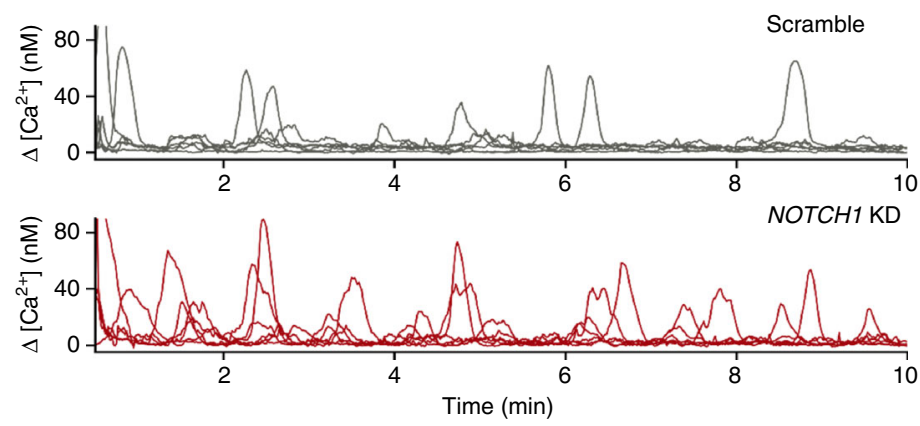

C

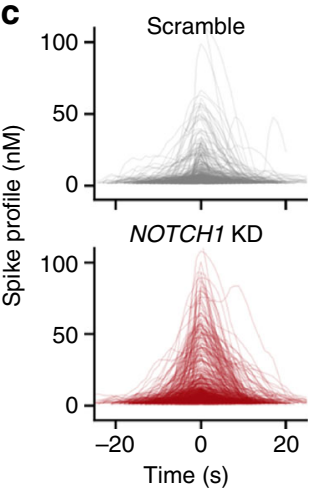

d
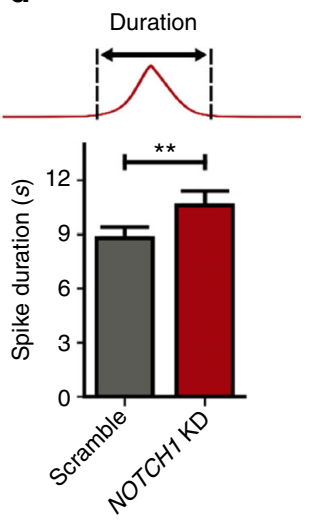

e
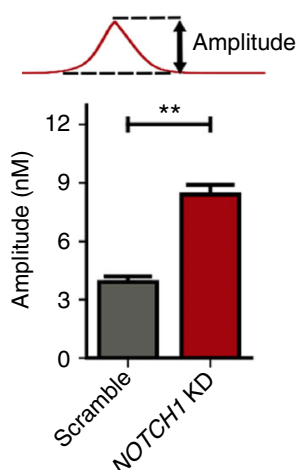

b

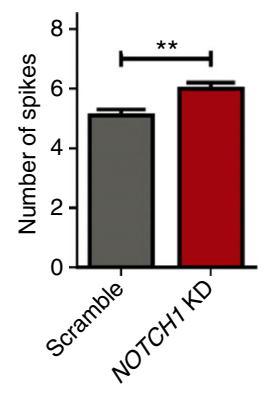

f g

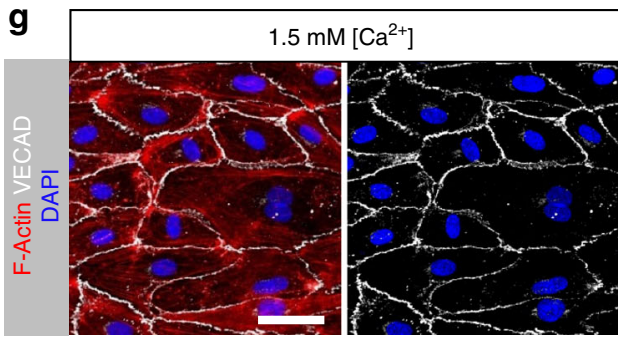

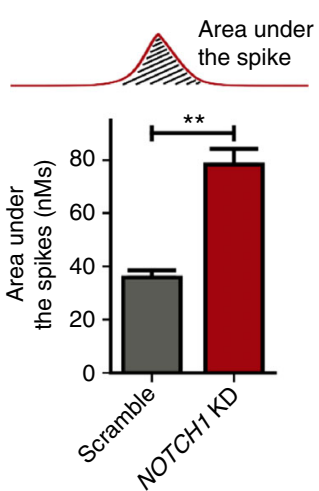
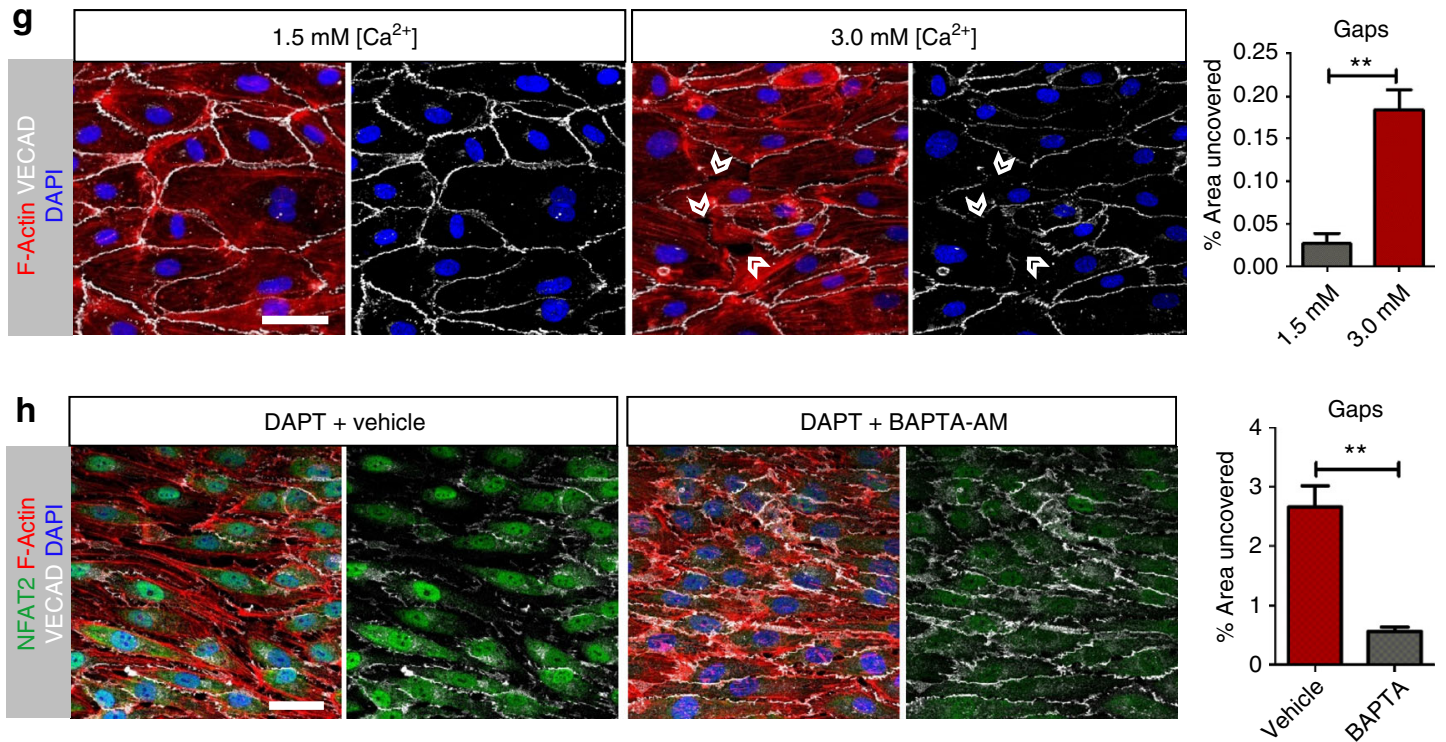

Fig. 7 Reduction in NOTCH1 increases $\mathrm{Ca}^{2+}$ signaling under flow. a Typical examples of $\mathrm{Ca}^{2+}$ responses using OGB-1-loaded HAECs after onset of flow at $t=0$ for Scramble (top) and NOTCH1 KD (bottom). b-f Evaluations correspond to data from more than 300 cells in each condition: Scramble ( $n=347$ ) and NOTCH1 KD $(n=339)$ obtained from three independent biological replicates for each condition. Graph bars represent mean \pm SEM. Significance was assessed by a Mann-Whitney U-test with ${ }^{\star \star} P<0.01$. b Average number of spikes in each responding cell is greater for NOTCH1 KD compared to Scramble. c Average $\mathrm{Ca}^{2+}$ spike plotted for each responding cell for Scramble and NOTCH1 KD. Peaks were aligned at $t=0 \mathrm{~s}$. d Average Ca ${ }^{2+}$ spike duration for each cell shows increased duration for NOTCH1 KD. e Average $\mathrm{Ca}^{2+}$ spike amplitude was larger for NOTCH1 KD. $\mathbf{f}$ Average area under the spike for each cell was significantly larger for NOTCH1 KD cells compared to Scramble. $\mathbf{g}$ Monolayers of HAECs were cultured in normal media with physiological levels of $\left[\mathrm{Ca}^{2+}\right](1.5 \mathrm{mM})$ or with $\mathrm{CaCl}_{2}$ added to increase extracellular $\left[\mathrm{Ca}^{2+}\right]$ to $3 \mathrm{mM}$ for $24 \mathrm{~h}$ under flow; scale bar $=20 \mu \mathrm{m}$. Gaps (marked in white arrow heads) were only observed in the HAEC monolayers cultured with high extracellular $\left[\mathrm{Ca}^{2+}\right]$. Gaps were quantified by measuring area uncovered to reveal an increase in gaps for monolayers cultured in higher extracellular $\left[\mathrm{Ca}^{2+}\right]$. Graph bars represent mean $\pm \mathrm{SEM}, n=5$. Significance was assessed by $T$-test ${ }^{\star \star} P<0.01$. $\mathbf{h}$ HAECs were flow-conditioned for $24 \mathrm{~h}$ then treated with BAPTA-AM or vehicle and subsequently returned to flow for an additional hour in the presence of DAPT; scale bar $=20 \mu \mathrm{m}$. Gaps were quantified by measuring the percent area uncovered to reveal that BAPTA treatment significantly improved junctional stability even in the absence of NOTCH1 signaling. For all imaging experiments, three biological replicates were used for each condition. Graph bars represent average \pm SEM. Significance was assessed by $T$-test ${ }^{\star *} P<0.01$ 
a

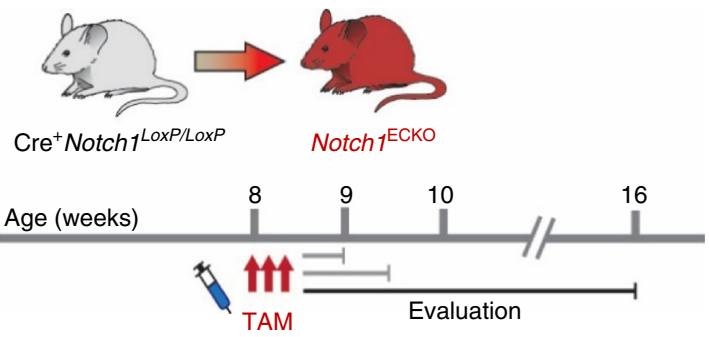

b

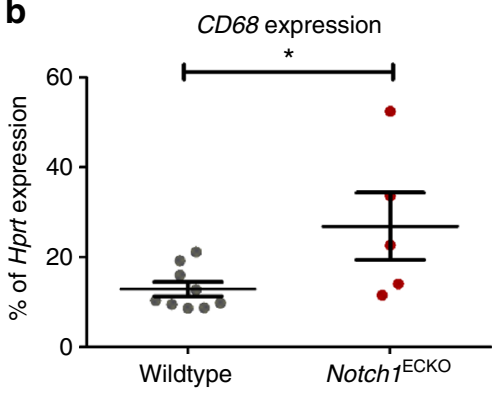

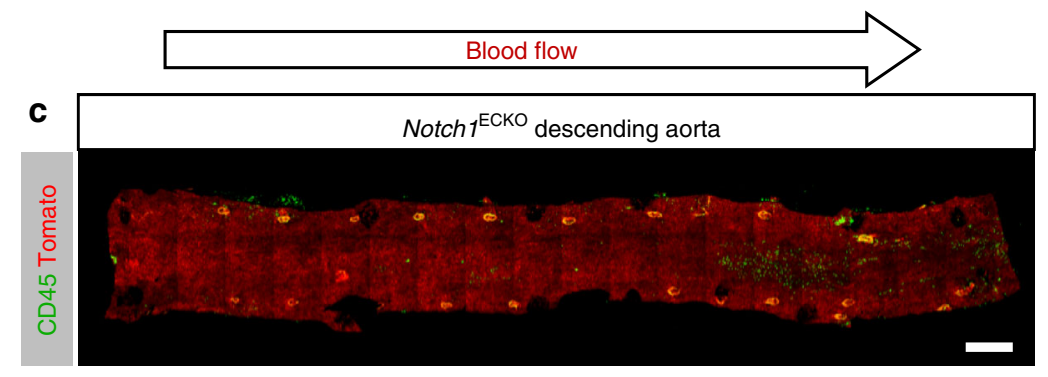
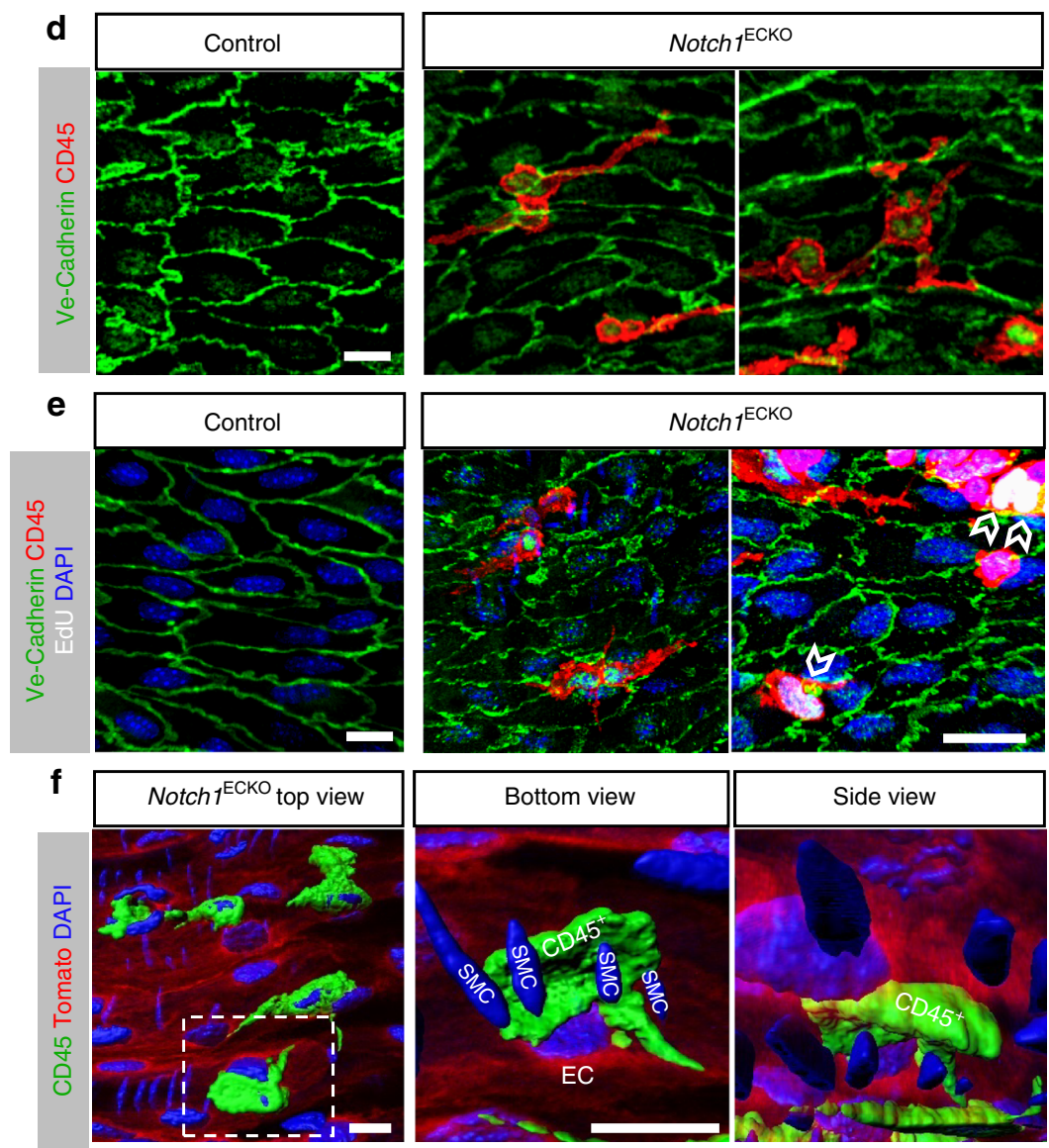

Fig. 8 In vivo deletion of endothelial Notch1 results in inflammatory cell recruitment. a Timeline for inducible deletion of endothelial Notch1 to indicate animal ages and evaluation time points. $\mathbf{b}$ Quantification of CD68 expression from the intima of descending aortae of adult WT mice $(n=9)$ and Notch $7^{\mathrm{ECKO}}$ mice $(n=5) 2$ weeks post tamoxifen by qPCR analysis. Graph bars represent average \pm SEM, $T$-test ${ }^{\star} P<0.01$. c Confocal tile scan of the descending aorta of Notch7 ${ }^{\mathrm{ECKO}}$ animal at 8 weeks post tamoxifen reveals the presence of $\mathrm{CD}_{4} 5^{+}$tdTomato $^{-}$cells embedded within the tdTomato ${ }^{+}$ endothelium. Scale bar $=500 \mu \mathrm{m}$. d Ve-Cadherin and CD45 staining of control ( $\mathrm{Cre}^{-}$Notch $^{\text {loxP/loxP }}$ ) and Notch $7^{\mathrm{ECKO}}$ descending aortae at 8 weeks post tamoxifen displays $\mathrm{CD} 45^{+}$inflammatory cells at higher magnification in the Notch $1{ }^{\mathrm{ECKO}}$ aorta; scale bar $=10 \mu \mathrm{m}$. e En face imaging of the endothelium by Ve-Cadherin staining in the descending aorta of control ( $\mathrm{Cre}^{-}$Notchl ${ }^{\text {loxP/loxP }}$ ) and Notch ${ }^{\mathrm{ECKO}}$ mice, both tamoxifen-injected and sacrificed after 8 weeks, shows proliferating $\left(\mathrm{EdU}^{+}\right) \mathrm{CD} 45^{+}$inflammatory cells (marked by open arrow heads) embedded within the endothelial layer of Notch $7^{\mathrm{ECKO}}$ mice; scale bars $=10 \mu \mathrm{m}$. $\mathbf{f}$ Three-dimensional surface render to visualize the spatial location of marked $\mathrm{CD} 45^{+}$cell relative to the endothelium (marked EC) and smooth muscle cells (marked SMC). Both bottom and side view reveal that the CD45 cell is locating above the smooth muscle cell layer. Scale bars $=10 \mu \mathrm{m}$ 
indicate that even in the adult, $\mathrm{NOTCH} 1$ is required to constantly maintain EPHRIN B2, and therefore, arterial identity.

Exploration of the mechanisms by which NOTCH1 signaling might rapidly and concurrently affect junctional stability, cell cycle, and elongation driven by flow were elusive until we noted a link between NOTCH1 and calcium homeostasis. Reduction of
NOTCH1 reveals an emerging signature of calcium-regulatory genes. These include CAMK2B a calcium/calmodulin-dependent protein kinase involved in multiple functions including regulation of $\mathrm{Ca}^{2+}$ release from the endoplasmic reticulum ${ }^{51}$; Apelin that, amongst other functions, was shown to regulate endoplasmic reticulum calcium channels (SERCA and ryanodine receptors) ${ }^{52}$; a
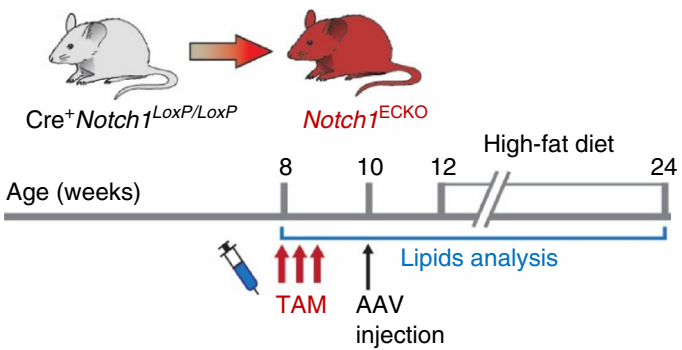

b

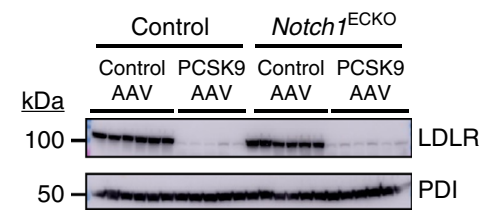

C $\widetilde{T}$

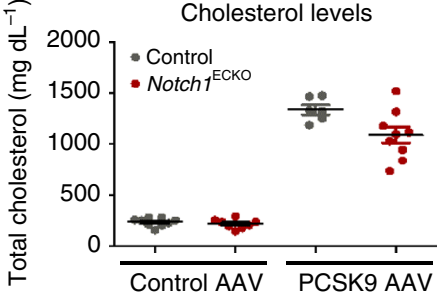

d

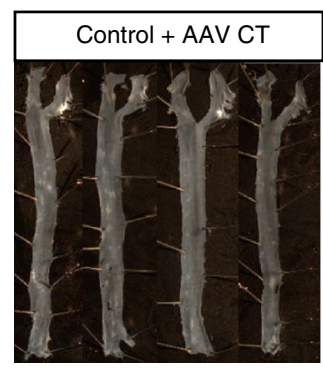

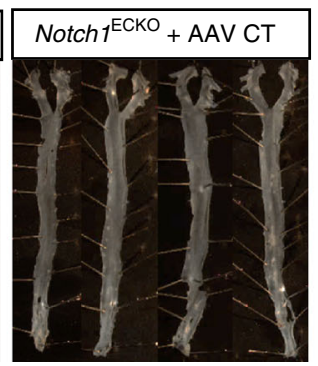
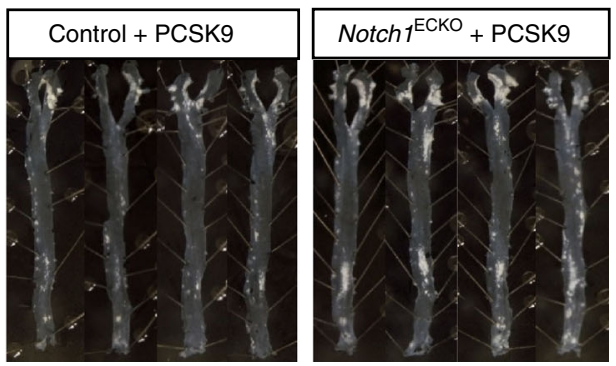

e

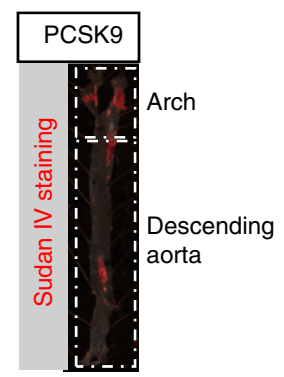

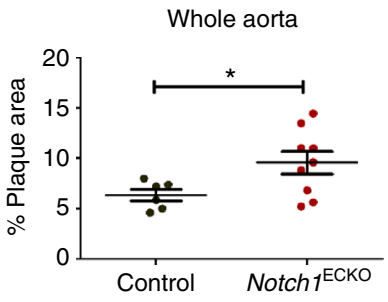
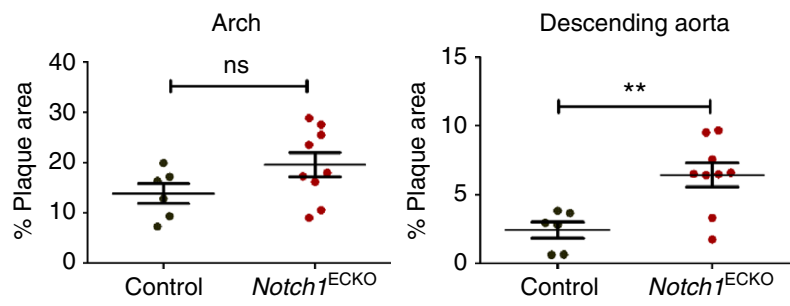
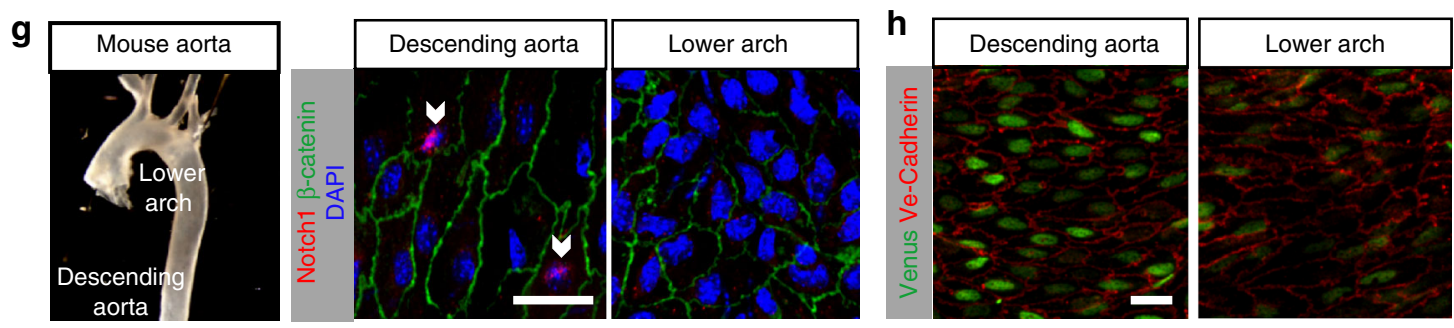

i
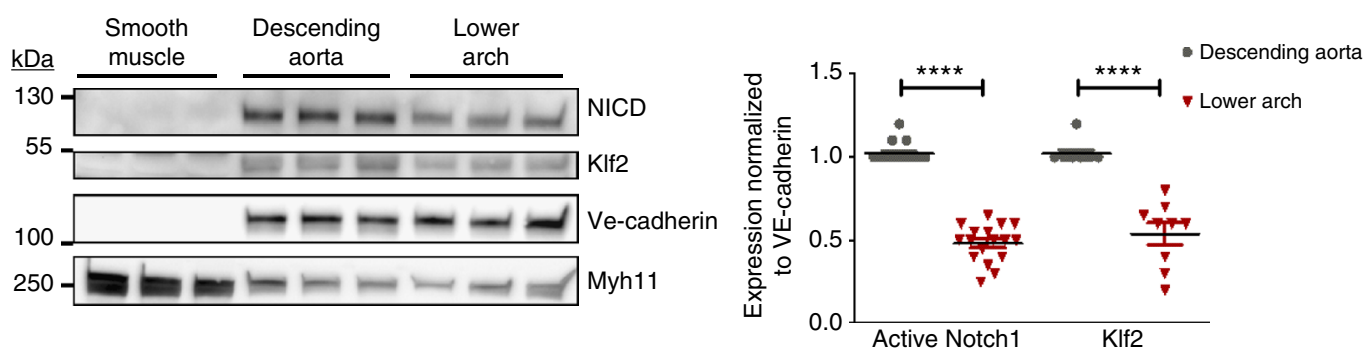
Connexin-37, involved in the formation of gap junctions that increase intercellular transport of calcium; and Aconitase, an enzyme that in addition to regulating tricarboxylic acid cycle also contributes to connexin hemichannel opening ${ }^{53}$. In total, 14 different transcripts associated with calcium regulation were increased upon NOTCH1 suppression (Supplementary Table 3). The transcriptional signature implies that NOTCH1 is involved in the rapid regulation of intracellular calcium, although it remains unclear whether these are direct NOTCH1 targets and whether these effects are mediated by a canonical NOTCH1 signaling event. Nonetheless suppression of NOTCH1 results in the increase of intracellular calcium signaling under flow. Importantly calcium is a powerful second messenger and slight increases in cytosolic levels have been associated with cell cycle regulation ${ }^{54-56}$, cell-cell junctions ${ }^{57}$, focal adhesion, and cytoskeletal changes ${ }^{58,59}$. The emerging concept from these findings is that NOTCH1 might be a modulator of intracellular calcium spikes and associated signaling events that are induced by flow ${ }^{60-62}$. In fact, recent work showed that $\mathrm{Ca}^{2+}$ oscillations were increased in tip cells vs. stalk cells during sprouting angiogenesis in zebrafish ${ }^{63}$. We propose that blood flow adjusts NOTCH1 to proportionally regulate $\mathrm{Ca}^{2+}$ signaling to ensure homeostasis.

Finally, we also showed that NOTCH1 signaling regulates several pro-inflammatory genes associated with atherosclerosis. The list included genes involved in recruitment of inflammatory cells such as CXCL2, ICAM1, CXCR4, and several CXCL ligands and interleukins (Supplementary Table 2). The list of transcripts reveals that $\mathrm{NOTCH} 1$ contributes to suppress endothelial binding to inflammatory cells and to enhance self-immunity. In the absence of NOTCH1, inflammatory cells incorporate within the endothelial layer and transmigrate across the intima. Of note, we find that VCAM is a direct target of NOTCH1 and it is suppressed rather than induced when NOTCH1 is reduced (Supplementary Table 2). The binding of macrophages is likely facilitated by intercellular gaps, as accumulation of fibrin most certainly enables binding and further spreading of these cells. These results are consistent with our finding that NOTCH1 haploinsufficiency in the context of a pro-atherogenic genetic background increases atherosclerosis in the aorta ${ }^{21}$ and further reveal that the protective role of NOTCH1 is particularly associated with the areas of laminar flow.

Overall, this study fills important gaps in our understanding of how blood flow controls endothelial responses. The data presented show that increases in laminar flow are sensed by NOTCH1 resulting in polarization and nuclear NICD translocation. Importantly, while endothelial cells are still able to sense flow, as per KLF2 increase, absence of NOTCH1 impairs their ability to remain mitotically quiescent and maintain junctional integrity. The pathophysiological consequences include increased susceptibility to atherosclerosis in areas considered to be atheroprotective. In this manner, NOTCH1 might be the point of conversion that interprets flow levels and controls responses to potential pathological conditions.

\section{Methods}

Cell culture. HUVECs (VEC Technologies \#HUVEC/T-75 Flask), HAECs (Lonza \#CC-2535 and ATCC \# PCS-100-011), and BAECs (isolated from bovine aortas) were cultured in complete MCDB-131 medium (VEC Technologies; MCDB131WOFBS) with $10 \%$ fetal bovine serum (Omega Scientific \#FB-11). All primary endothelial cells were checked for mycoplasma and cultured on gelatin (Fisher Scientific \# NC9369923) coated dishes in humidified incubator at $37^{\circ} \mathrm{C}$ and $5 \%$ $\mathrm{CO}_{2}$ and used between passages 4 and 9 .

Shear stress. Endothelial cells were seeded in ibidi $\mu$-Slide $y$-shaped ibiTreat chambers (ibidi \#80126) or $\mu$-Slide I 0.4 Luer ibiTreat chambers (ibidi \#80176) and unidirectional flow was applied after confluent monolayer reached using the ibidi pump system (ibidi \#10902). For RNA and protein lysis, laminar shear stress was applied using a modified cone and plate device ${ }^{64}$ consisting of a computerized stepper motor UMD-17 (Arcus Technology \#DMX-UMD-17-2) and a $1^{\circ}$ tapered stainless steel cone set at $700 \mu \mathrm{m}$ height above the plate. The flow devices were maintained in $37^{\circ} \mathrm{C}$ incubator with $5 \% \mathrm{CO}_{2}$. Confluent endothelial monolayers were grown on gelatin-coated six-well plates (Falcon \#08-772-1B) in complete MCDB-131 media (VEC Technologies \# MCDB131-WOFBS) plus 10\% FBS (Omega Scientific \#FB-11) containing 4\% dextran (Sigma-Aldrich \#31392). Monolayers were subjected to unidirectional constant laminar flow, pulsatile laminar flow (termed atheroprotective), or disturbed flow ${ }^{15}$ for set time points before lysates were harvested, at which point the center of each well was removed with a cell scraper (BD Falcon \#35-3085) before lysis buffer was added. Static monolayers used the same dextran used the same dextran-containing media and were cultured alongside flow-treated monolayers. For pre-aligned treatments, DMSO or DAPT were added after $48 \mathrm{~h}$ of flow treatment directly to culture medium within the well.

Immunofluorescence and imaging. Cells were fixed in flow chambers with $2 \%$ PFA or methanol (Sigma-Aldrich \#322415) for $10 \mathrm{~min}$ followed permeabilized with $0.1 \%$ Triton X-100 (Sigma Aldrich \#X100) in $1 \times$ PBS and blocked for $2 \mathrm{~h}$ with $5 \%$ Normal donkey serum (Jackson Immuno Research Laboratories \#017-000-121) in 1X PBS. Primary antibodies were incubated overnight at $4{ }^{\circ} \mathrm{C}$ in blocking buffer and secondary antibodies applied for $2 \mathrm{~h}$ at room temperature. To harvest mouse aortas for imaging, the animals were injected with $6.5 \mathrm{mg}$ methacholine chloride (MP Biomedicals \# 0219023105) dissolved in 1× PBS to relax smooth muscle and then perfusion-fixed with $2 \%$ PFA via left ventricle. The aorta was subsequently dissected from the spine, adventitia removed, cut longitudinally, and then pinned opened in silicone-coated dishes for staining after further fixation, permeabilization, and block. The aortas were mounted en face using ProLong Gold Antifade Mountant (Fisher Scientific \#P36930). Primary antibodies: anti- $\beta$-catenin rabbit polyclonal (1:200; Sigma \#C2206), anti- $\beta$-catenin goat polyclonal (C-18) (1:200; Santa Cruz Biotechnology \#sc-1496), anti-NOTCH1 (D1E11) rabbit monoclonal (1:200; Cell Signaling \#3608), anti-NOTCH1 ECD mouse monoclonal (1:200; BD Biosciences \#MHN1-519), anti-VE-cadherin goat polyclonal (Santa Cruz Biotechnology \#sc-6458), anti-Giantin mouse monoclonal (1:200; Abcam \#ab37266), anti-acetylated tubulin mouse monoclonal (1:200; Sigma \#T7451), anti-GFP chicken polyclonal (1:200; Abcam \#ab13970), anti-ERG (EPR3864) rabbit monoclonal (1:200; Abcam \#ab92513), anti-mouse CD45 Clone 30-F11 rat monoclonal (1:200; BD Biosciences \#550539), and anti-NFAT2/NFATC1 rabbit polyclonal (1:200; Novus Biologicals \#NB100-56732). Fluorescently tagged secondary antibodies were purchased from Invitrogen, applied at 1:400 dilution and in some

Fig. 9 Loss of endothelial Notch1 augments atherosclerotic plaque burden in a model of hypercholesterolemia. a Timeline for mouse model of hypercholesterolemia. b Quantification of liver LDLR protein levels by immunoblot to confirm PCSK9 activity in PCSK9-AAV-injected animals compared to control-AAV-injected animals at time of harvest (PDI used as loading control). Each lane corresponds to one animal. c Circulating cholesterol levels at time of evaluation (12 weeks after AAV injection) ( $n=6-11$ per group, each dot corresponding to one animal). Indicated are individual cholesterol levels in each group. d Representative aortas from each of the four groups: Control + AAV CT, Notch $7^{\mathrm{ECKO}}+\mathrm{AAV}$ CT, Control + PCSK9, and Notch7 ECKO + PCSK9. Control animals were $\mathrm{Cre}^{+}$tdTomato reporter. e Example of aorta stained with Sudan IV for quantification of atherosclerotic plaque area and identification of the arch and descending aorta regions. $\mathbf{f}$ Quantification of plaque burden in the whole aorta, arch, and descending aorta was determined by calculating the percent of aortic surface area covered by atherosclerotic lesions in each group ( $n=6$ for Control and $n=9$ for Notch7 ${ }^{\mathrm{ECKO}}$ ). Each point represents the lesion area per mouse. The mean area for each group of mice is indicated by the horizontal bars. $T$-test ${ }^{\star} P<0.05,{ }^{\star \star} P<0.01$, ns $=$ not significant. $\mathbf{g}$ En face imaging of wild-type (C57BL/6) adult mouse aorta reveals enhanced nuclear presence of Notch1 (red) in endothelial cells of the descending aorta compared to endothelial cells of the lower arch (representative images of $n=6$ ). Scale bar $=20 \mu \mathrm{m}$. $\mathbf{h}$ Venus Notch reporter mouse aorta imaged en face to assess Notch signaling comparing the descending aorta to the arch (representative images of $n=3$ ). Scale bar $=20 \mu \mathrm{m}$. i Protein lysates from bovine aorta (descending aorta, lower arch and smooth muscle) were analyzed by immunoblot for expression of Notch1 intracellular domain (NICD) and KIf2 using Ve-Cadherin as loading control. Each lane shows isolation from a distinct biological replicate. NICD levels trend with KIf2 expression level differences between the descending aorta and lower arch (data from nine technical replicates isolated from four biological replicates are shown on graph, $T$-test ${ }^{\star \star \star \star} P<0.0001$ ) 
instances used in combination with Texas Red-X Phalloidin (1:40; Life Technologies \# T7471) and DAPI (1:1000; Thermo Scientific \#62248). For mouse aorta en face imaging of Notch1, Tyramide Signal Amplification (TSA) kit (ThermoFisher Scientific \#T20934) was used and followed according to the manufacturer's supplied protocol. The images were captured with $\times 10, \times 20$, or $\times 40$ objective on a Zeiss LSM710 or LSM880 confocal microscope with ZEN software (Carl Zeiss Microscopy) for acquisition. Image processing and quantification of parameters was performed with Imaris software (Bitplane) or ImageJ.

\section{Transfection and $\boldsymbol{\gamma}$-secretase inhibition. Confluent monolayers of endothelial} cells were transfected with stealth RNAi targeting NOTCH1

(NM_017617.3_Stealth_775: Sense-GAC GAU UGU CCA GGA AAC AAC UGC A and Antisense-UGC AGU UGU UUC CUG GAC AAU CGU C) or control Stealth RNAi ${ }^{\mathrm{mw}}$ siRNA Negative Control, Med GC (Invitrogen \#12935300) using siPORT Amine transfection agent (Invitrogen \#AM4503) in Opti-MEM ${ }^{\circledR}$ Reduced Serum Medium (Gibco \# 31985070) without antibiotics. For improved efficiency, the cells were transfected twice with $24 \mathrm{~h}$ of recovery in between; cultures were then used for experiments within $12 \mathrm{~h}$ after the second transfection. For in vitro NOTCH signaling experiments, Cignal RBP-Jk Reporter (GFP) kit (QIAGEN \#CCS-1014G) was used by transfecting HUVECs or HAECs according to the manufacturer's guidelines. For experiments involving $\gamma$-secretase inhibition, 10 or $50 \mu \mathrm{M}$ InSolution $\gamma$-secretase inhibitor IX (EMD Millipore \#565784) or equivalent volume of dimethyl sulfoxide (DMSO) (Hybri-Max ${ }^{\mathrm{mm}}$, sterile-filtered, BioReagent $\geq 99.7 \%$ Sigma-Aldrich \#D2650) was applied to the cell culture medium for the duration of the experiment.

Western blot analysis. Endothelial cells were lysed in modified RIPA buffer containing $1 \%$ Triton X-100 and $10 \%$ SDS. For all in vitro cultures, the endothelial cell monolayers in 6-well plates were lysed after removing the center region using cell scraper (BD Falcon \#35-3085) and washing with 1X HBSS (Corning \#21-022$\mathrm{CV}$ ) to avoid the region not subjected to uniform shear stress. Flow-treated cultures and static controls were handled equivalently. Proteins were separated by SDSPAGE gradient (4-20\%) gel and transferred onto nitrocellulose membranes and incubated overnight at $4{ }^{\circ} \mathrm{C}$ with primary antibodies. Primary antibodies: anti-VeCadherin rabbit polyclonal (1:1000; Cell Signaling \#2158), anti-NOTCH1 (D1E11) rabbit monoclonal (1:1000; Cell Signaling \#3608), Cleaved NOTCH1 (Val1744) (D3B8) rabbit monoclonal (1:500; Cell Signaling Technology \#4147), anti-Klf2 rabbit polyclonal (1:500; EMD Millipore \#09-820), anti-Cyclin D1 rabbit monoclonal (1:500; Abcam \#ab16663), anti-gamma H2A.X (phospho S139) rabbit polyclonal (1:1000; Abcam \#ab11174), anti-smooth muscle Myosin heavy chain 11 rabbit polyclonal (1:1000; Abcam \#ab53219), anti-gamma Tubulin rabbit polyclonal (1:2000; Abcam \#ab11321), anti-LDL Receptor rabbit polyclonal (1:1000; Cayman \#10007665), and anti-PDI rabbit monoclonal (1:10,000; Cell Signaling \#3501). HRP-conjugated secondary antibodies $(1: 10,000)$ were applied in species dependent manner at room temperature for $1 \mathrm{~h}$. Immuno-complexes were detected by enhanced chemiluminescence with SuperSignal ${ }^{\text {mit }}$ West Femto Maximum Sensitivity Substrate (ThermoFisher Scientific \#34095) using ChemiDoc XRS + Molecular Imager (Bio-Rad Laboratories). Quantification of bands by densitometry analysis was performed using ImageLab Software (Bio-Rad Laboratories).

MRI flow mapping. The Y-slide was positioned in a $40 \mathrm{~mm}$ i.d. $400 \mathrm{MHz} 1 \mathrm{H}$ Millipede vertical imaging probe within $9.4 \mathrm{~T}$ Varian microimaging system. Flow was imaged using a spin-echo multi-slice (SEMS) pulse sequence modified for phase-contrast MRI velocimetry ${ }^{65}$. Briefly, all gradients in the SEMS pulse sequence, except for the phase encoding gradient, were appended with flow-compensation gradients. Bipolar, trapezoidal, FW gradients were added in the $x, y$ and $z$ directions to select the gradient first moment, M1. The residual phase accumulation from each F.W. gradient is proportional to the fluid velocity in the respective direction when M1 is properly chosen to avoid phase wrapping ${ }^{66}$. In the case of no flow, phase accumulation of static nuclear spins resulting from a gradient value of $+\mathrm{M} 1$ and $-\mathrm{M} 1$ is neutralized, resulting in a zero net phase accumulation. Fluid flow speeds were calculated using the $x, y$ and $z$ velocity components. Flow vector maps were generated from the $x$ and $y$ velocity components. To compensate for directional fluid velocity bias, the $y$-slide was imaged, physically rotated $180^{\circ}$ about the $x$ axis inside the bore of the magnet, and imaged again. The initial position and $180^{\circ}$ rotated F.W. images were averaged together to generate the final result. To account for gradient imperfections, each of these images was generated by subtracting a flow-weighted image of the slide in the absence of flow. Corresponding shear maps were determined using the equation: $\tau_{w}=\frac{6 \mu Q}{b h^{2}}$ where $\tau_{w}$ is the shear stress at the endothelial cell monolayer, $\mu$ is the dynamic viscosity of cell culture media at $37^{\circ} \mathrm{C}$, $Q$ is the volumetric flow rate through a voxel, $b$ is the width of a voxel, and $h$ is the height of the ibidi chamber. This equation is derived from the Navier-Stokes equation ${ }^{67}$, given a condition of laminar flow between two infinitely wide parallel plates.

NOTCH1 protein polarization kinetics. To evaluate the spatial distribution of NOTCH1 intracellular domain (ICD) and extracellular domain (ECD), after flow alignment for $48 \mathrm{~h}$, HAECs were exposed to reverse flow direction for different periods of time. For each time point of reverse flow, at least three biological replicates were used from which $n \geq 116$ individual cells were measured. To estimate the concentrations of NOTCH1 ICD and ECD, ImageJ was used to calculate the fluorescence along the cell body length and parallel to the flow axis. Cell bodies that were partially visible were discarded. Using the center of the nucleus as a point of reference, the distance from both ends of the cell body to the center of the nucleus were normalized to 1 , allowing the evaluation of the distribution of ICD and ECD as a function of time and position along the cell body. To evaluate the concentration of ICD and ECD in each side of the cell bodies with respect to the nucleus, we removed the background fluorescence and evaluated the area under the curve of fluorescence in each side separately and normalized by both areas. To evaluate the polarization kinetics, the average concentration of ICD and ECD at each time point analyzed was then fitted with an exponential function and measured the diffusion rate as the exponential decay's characteristic time.

Intracellular calcium imaging. HAECs were plated on y-slide (ibidi \#80126) and after reaching confluence, cells were treated with $15 \mu \mathrm{M}$ Oregon Green 488 BAPTA-1-AM ester (OGB-1-AM) (Thermo Fisher Scientific \#O6806). The AM ester can freely pass the cell membrane; but once inside, the AM is cleaved by esterases leaving OGB-1 trapped in the cell. OGB-1-AM was reconstituted in Pluronic F-127 (Invitrogen \#P-3000MP) and diluted in phenol-red-free MCDB131 (VEC Technologies \#MCDB-131 WOFBSPR) with 10\% FBS (Fisher Scientific, Cat. No. MT 21-023-CV). After 20-min incubation with OGB-1-AM, the cells were washed with $1 \mu \mathrm{g} / \mathrm{mL}$ Hoescht (Thermo Fisher Scientific \#PI-62249) diluted in MCDB-131 (MCDB-131 WOFBSPR) and incubated for an additional $20 \mathrm{~min}$. Slides were then connected to the yellow/green perfusion set (ibidi \#10963) modified with non-permeable tubing containing $13 \mathrm{~mL}$ of conditioned MCDB-131 (MCDB-131 WOFBSPR, phenol red free). All steps were completed with minimal light exposure. After beginning flow (20 dynes $\left.\mathrm{cm}^{-2}\right)$, fluorescence images were acquired using Zeiss Observer Z1 with Colibri 7 light source, ORCA-Flash4.0 camera (Hamamatsu \#C13440-20CU) and ZEN 2 (blue edition) software. Images were acquired once every second with a $50 \mathrm{~ms}$ exposure. After $10 \mathrm{~min}$ of imaging the flow was stopped. To obtain $F_{\max }$, the calcium ionophore ionomycin (Life Technologies \#I24222) was added to the perfusion set to obtain a final concentration of $1.5 \mu \mathrm{M}$. Flow was restarted, and images were captured every second for $3 \mathrm{~min}$. At the conclusion of $3 \mathrm{~min}$, the flow was stopped and media in perfusion reservoirs removed. To obtain $F_{\min }$, conditioned HEPES buffered EDTA solution (20 mM EDTA, $120 \mathrm{mM} \mathrm{NaCl}, 5 \mathrm{mM} \mathrm{KCl}$, and $10 \mathrm{mM}$ HEPES) was added, the flow was restarted and images were captured every second for $3 \mathrm{~min}$.

Calcium imaging analysis. To detect and segment cells, all frames of the calcium imaging videos were averaged and Otsu's threshold applied. To remove photo bleaching from the calcium imaging, a non-linear fitting of the signal was used to subtract the slow decay. Because the signals often consisted of several wide peaks that represented a reasonable portion of the time series, an asymmetric loss function was used to penalize overestimations more than underestimations. The fitted model combined a second degree polynomial with an exponential decay. The asymmetric loss function used in this non-linear fitting was

$$
L(y, \hat{y})=(y-\hat{y})^{2}(\operatorname{sign}(y-\hat{y})+\alpha)^{4},
$$

where $y$ represents the predicted values by the fitted model, $\hat{y}$ represents the actual measured values, and $\alpha>0$ is a meta-parameter optimized for each sample (for most replicates, $\alpha \approx 0.8$ ). The optimization was performed by the Levenberg-Marquard algorithm (lmfit implementation) with the linear coefficient and the exponential characteristic time both constrained to negative values. For more robust fitting, we also added an L2 regularization term on the coefficient of second degree term. After removing the photo bleaching, the calcium concentration was estimated by using the formula $F_{\mathrm{Ca}}(t)=K_{\mathrm{d}}\left(F(t)-F_{\min }\right) /\left(F_{\max }-F(t)\right)$, where $F(t)$ is the fluorescence as a function of time ${ }^{68}, K_{\mathrm{d}}=170 \mathrm{nM}$ is the dissociation constant of OGB-1, and $F_{\max }$ and $F_{\min }$ were both determined for each cell separately (see above). Figure $7 \mathrm{a}$ shows representative time series plots of the calcium concentration $F_{\mathrm{Ca}}(t)$ for control cells and for Notch1 KD cells. To detect spikes, a threshold for each replicate was defined in order to select spikes that were two standard deviations above the noise and lasted for at least three seconds. Spike duration for each cell was calculated by measuring how long the calcium concentration $F_{\mathrm{Ca}}(t)$ was above a set threshold value. To evaluate the area under the spike, we subtracted from $F_{\mathrm{Ca}}(t)$ its minimum and evaluated the total area under the curve. Spike amplitude was measured as the highest calcium concentration, with respect to the baseline, achieved in each spike for each cell.

Intracellular calcium blocking experiments. HAECs were plated on a Y-shaped slide (ibidi \#80126). After reaching confluence, cells were exposed to flow (20 dynes $\mathrm{cm}^{-2}$ ) for $48 \mathrm{~h}$ using red perfusion set (ibidi \#10962) containing $13 \mathrm{~mL}$ of conditioned MCDB-131 with $10 \%$ FBS. After $48 \mathrm{~h}$, the cells were treated with $20 \mu \mathrm{M}$ BAPTA-AM (Life Technologies \#B6769) for $20 \mathrm{~min}$. BAPTA-AM was reconstituted in Pluronic F-127 (Invitrogen \#P-3000MP) and diluted in MCDB-131 (VEC Technologies \#MCDB-131 WOFBS) with 10\% FBS (Fisher Scientific, \#MT 21-023-CV). After a 20-min incubation with BAPTA-AM, slides were reconnected to perfusion sets and exposed to flow $\left(20\right.$ dynes $\left.\mathrm{cm}^{-2}\right)$ for $1 \mathrm{~h}$ in the presence of 50 
$\mu \mathrm{M}$ DAPT or equivalent volume of DMSO. After $1 \mathrm{~h}$, the slides were removed and fixed with $2 \%$ PFA and subsequently imaged for confocal microscopy.

Transcriptional analysis. Endothelial RNA-enriched fractions from mouse aortae were harvested from descending aortae after careful dissection under a dissecting microscope (Zeiss Stereo Discovery V12). The lumen of the aorta was subsequently flushed with $100 \mu \mathrm{L}$ of RLT lysis buffer (QIAGEN \#79216) containing $1 \% \beta$-mercaptoethanol (MP Biomedicals \#0219483483) using an insulin syringe $(\mathrm{BD} \# 328431)^{69}$. Endothelial cell monolayers under flow were also lysed using RLT lysis buffer with $1 \% \beta$-mercaptoethanol after the center region was removed with cell scraper (BD Falcon \#35-3085) and washed in 1X HBSS. Total RNA from cell culture or mouse aortae was purified using the RNeasy Mini kit (QIAGEN \#74106). Complementary DNA synthesis was performed with Superscript III reverse transcription First-Strand synthesis kit (Invitrogen \#18080051) using oligodT primers. qRT-PCR was performed using primers designed for human or mouse targets, each reaction was run in duplicate and gene expression was normalized with the housekeeping gene (HPRT) and relative expression calculated using the $\Delta \Delta \mathrm{Ct}$ method. Primer sequences for mouse targets: Hprt 5'-CTGGTTAAGCAGTACAGCCCCAA-3' (forward) and 5'-CGAGAGGTCCTTTTCACCAGC-3' (reverse); Notch1 5'-CCCTTGCTCTGCCTAACGC-3' (forward) and 5'-GGAG TCCTGGCATCGTTGG-3' (reverse); Cd68 5' - GACCTACATCAGAGCCC GAGT-3' (forward) and 5'-CGCCATGAATGTCCACTG-3' (reverse). Primers sequences for human targets: HPRT 5'-GCCCTGGCGTCGTGATTAGT-3' (forward) 5'-AGCAAGACGTTCAGTCCTGTC-3' (reverse); NOTCH1 5' -ACTGTGAGGACCTGGTGGAC-3' (forward) 5'-TTGTAGGTGTTGGGGAGGTC-3' (reverse); HES1 5' -TCA ACA CGA CAC CGG ATA AA-3' (forward) 5'-TCA GCT GGC TCA GAC TTT CA-3' (reverse); KLF2 5'-CATCTGAAGGCGCATCTG-3'

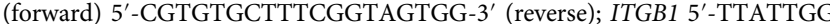
CCTTGCATTACTGCT-3' (forward) 5'-CCACAGTTGTTACGGCACTCT-3' (reverse); PECAM1 5'-GAAACCATGCAATGAAACCA-3' (forward) 5'-GACAG CTTTCCGGACTTCAC-3' (reverse); ICAM1 5'-ACC GGA AGG TGT ATG AAC TG-3' (forward) 5'-AGC GTA GGG TAA GGT TCT TG-3' (reverse); IL8 5'-AAG AAA CCA CCG GAA GGA AC-3' (forward) $5^{\prime}$-ACT CCT TGG CAA AAC TGC AC-3' (reverse); CCL2 5'-CAG CCA GAT GCA ATC AAT GCC-3' (forward) 5'TGG AAT CCT GAA CCC ACT TCT-3' (reverse). Primer sets were synthesized by Integrated DNA Technologies, Inc.

NanoString transcriptional analysis. For NanoString gene expression analysis, probe sequences for NOTCH1, HES1, NRARP, FABP4, EFNB2, KLF2, KLF4, and CCND1 were custom designed and manufactured by NanoString Technologies Inc., including sequences of 4 housekeeping genes (HPRT1, TLK2, TUBG1, and USP39) for data normalization, as well as negative and positive controls. Validation of RNA sample quality was done using the BioAnalyzer (Agilent Technologies). HAECs were used for all sample conditions and each culture condition was performed with $n=5$. The samples were loaded at $100 \mathrm{ng}$ mRNA per well and data collected as absolute counts; detectable levels of non-specific binding (background) were measured by six negative controls and the means plus two standard deviations were subtracted from each count. Analysis of the nCounter Gene Expression CodeSet was performed via nSolver analysis software (NanoString Technologies, Inc.) to calculate fold change expression.

Transcriptome analysis. Microarray results used for the analysis were deposited in the National Center for Biotechnology Information Gene Expression Omnibus database under accession no. GSE72633. HAECs were transfected in triplicate with stealth RNAi targeting NOTCH1 (NM_017617.3_Stealth_775) or control duplexes (Invitrogen) using siPORT Amine (Ambion) as the transfection agent. Cells were transfected twice with $24 \mathrm{~h}$ of recovery between, cultures were then used for experiments $24 \mathrm{~h}$ after the second transfection. For each siRNA condition, three biological replicates were performed and evaluated. Total RNA samples with RNA integrity numbers (RIN) of 7.0 or higher were hybridized to Illumina Human HT12 v4 Expression BeadChips (San Diego, CA), which contained 48,804 expression and 786 control probes. Differential gene expression analyses to compare cells transfected with siRNA against NOTCH1 or scrambled siRNA was performed using the limma package in $\mathrm{R}$ (v2.13.0) with non-parametric background correction followed by quantile normalization. Calculated $P$-values from the moderated $t$ statistics were adjusted using the Benjamini-Hochberg method for multiple testing. Microarray probes with adjusted values of $P<0.01$ (1\% FDR) and fold change $>$ $50 \%$ were considered differentially expressed. For visualization of the differential gene expression $\log$-2-fold change was plotted against $-\log 10$ of $P$-value using $\mathrm{R}$ and points were color coded based on gene ontology and defined fold change cut-offs.

Lipid analysis. Total plasma cholesterol was determined using an enzymatic, colorimetric assay kit (Infinity Cholesterol; Thermo Fisher), according to manufacturer's instructions. Briefly, plasma was isolated by centrifugation and stored at $-80^{\circ} \mathrm{C}$ until assaying. For each assay plate, a standard curve ranging between 0 and $300 \mathrm{mg} \mathrm{dL}^{-1}$ was run. A volume of $10 \mu \mathrm{L}$ of standard or sample was pipetted onto a 96-well plate and $200 \mu \mathrm{L}$ of working reagent (Infinity Cholesterol) added. The plates were covered, incubated for $15 \mathrm{~min}$, and read at $550 \mathrm{nM}$ (with a reference wavelength of $650 \mathrm{nM})$. All samples were performed in triplicate, where any replicates differing by $5 \%$ or more were re-assayed.

Animal experiments. Animal protocols were reviewed and approved by the UCLA Institutional Animal Care and Use Committee. C57BL/6J mice were purchased from Jackson Laboratory (stock \#000664) to expand the colonies, both female and male adult mice were used in all the experiments. For inducible endothelial deletion of Notch1, Cdh5(PAC)-CreERT2 (Tg(Cdh5-cre/ERT2)1Rha) mice, Notch $1^{\text {tm2Rko }}$ loxp line were crossed with R26RTd Cre reporter lines (Gt(ROSA)26Sor ${ }^{\mathrm{tm} 14(\mathrm{CAG}}$ tdTomato)Hze $)^{70}$. Eight-week-old mice received intraperitoneal injections of tamoxifen (1 mg per mouse; MP Biomedicals \#02156738) for three consecutive days and then sacrificed as indicated. CBF:H2B-Venus reporter mice ( $\mathrm{Tg}(\mathrm{Cp}-\mathrm{HIST} 1 \mathrm{H} 2 \mathrm{BB} /$ Venus) $47 \mathrm{Hadj} / \mathrm{J}$ ) were purchased from the Jackson Laboratory (stock \#020942) and used for imaging experiments. To quantify proliferation, EdU (5-ethynyl-2'deoxyuridine) (Invitrogen \#A10044) was applied via intraperitoneal injection (1 mg in $1 \times$ PBS warmed to $37^{\circ} \mathrm{C}$ ) $2 \mathrm{~h}$ before animal sacrifice. Click-iT ${ }^{\circledR}$ EdU Alexa Fluor ${ }^{\circledR} 488$ Imaging Kit (Invitrogen \# C10337) was used on 2\% PFA fixed aorta and followed according to the manufacturer's protocol.

For the mouse hypercholesterolemia study, AAV-PCSK9 and control was a gift Dr. H. Jo ${ }^{38}$. Two weeks after tamoxifen injections, the mice were injected with AAV-PCSK9 $\left(1 \times 10^{11} \mathrm{VG}\right)$ or control AAV via tail vein and fed a high-fat diet (16\% fat and $1.25 \%$ cholesterol, Research Diets \#D12336) for 3 months. Blood was collected every 2 weeks via retro-orbital bleed to assess circulating lipid levels. At the time of harvest, livers were isolated to obtain protein lysates and the aortas were subsequently perfused fixed and stored in $4 \%$ PFA $+7.5 \%$ sucrose. Aortas were then pinned on black wax, rinsed in $70 \%$ ethanol for $5 \mathrm{~min}$, stained with Sudan IV solution for $15 \mathrm{~min}$, and de-stained in $80 \%$ ethanol. Images were taken using Zeiss Stereo Discovery V12 microscope with Zeiss Axiocam HRC and Axiovision software. To automate the aortic plaque quantification, ImagePro Premier software (Media Cybernetics) was used to apply Spatial Calibration and Smart Segmentation tools to determine the total aorta area and plaque area. The plaque area was quantified as a percent of the total aorta and as a percent for the arch, defined by the arch and $3 \mathrm{~mm}$ from the arch bifurcation cut, and the descending aorta, defined by the remaining aorta length.

Mouse experiments were conducted in accordance with UCLA Department of Laboratory Animal Medicine's Animal Research Committee guidelines.

Bovine aortas were obtained from Sierra for Medical Science (Whittier, CA) and from animals that were sacrificed for purposes other than experimental.

Statistics, sample size, and blinded analysis. Statistical analyses were included in almost all of the experiments with the few exceptions of qualitative image analysis. Dr. David Elashoff (biostatistics core at UCLA) provided oversight. Determination of variance between groups, parametric, or non-parametric analysis was assessed and either $t$-test or Mann-Whitney was applied to determine significance. Most analysis were performed using Prism software (GraphPad Software). $P<0.05$ was considered significant and reported to the graphs. Data are represented as mean \pm SEM. Differential gene expression analyses to compare cells transfected with siRNA for NOTCH1 or Scramble was performed using the limma package in R (v2.13.0). Calculated $P$-values from the moderate Student's $t$-statistics were adjusted using the Benjamini-Hochberg method for multiple testing. Sample size and appropriate number of biological replicates has been discussed with our biostatistician for animals, imaging, and transcriptional/protein levels.

In multiple cases, collaborators performed the analysis of data in a manner that was blinded (cholesterol analysis, image analysis, atherosclerotic plaque burden).

Data availability. The data set for the gene microarray analysis on HAECs treated with siNOTCH1 or scramble was deposited in the National Center for Biotechnology Information Gene Expression Omnibus database under accession number GSE72633. The rest of the data is available from the authors upon reasonable request.

Received: 28 November 2016 Accepted: 13 October 2017 Published online: 20 November 2017

\section{References}

1. Hoffman, B. D., Grashoff, C. \& Schwartz, M. A. Dynamic molecular processes mediate cellular mechanotransduction. Nature 475, 316-323 (2011)

2. Davies, P. F. Hemodynamic shear stress and the endothelium in cardiovascular pathophysiology. Nat. Clin. Pract. Cardiovasc. Med. 6, 16-26 (2008).

3. Baeyens, N., Bandyopadhyay, C., Coon, B. G., Yun, S. \& Schwartz, M. A. Endothelial fluid shear stress sensing in vascular health and disease. J. Clin. Invest. 126, 821-828 (2016).

4. Garcia-Cardena, G., Commander, J., Anderson, K. R., Blackman, B. R. \& Gimbrone, M. A. Biomechanical activation of vascular endothelium as a 
determinant of its functional phenotype. Proc. Natl Acad. Sci. USA 98, 4478-4485 (2001).

5. McCue, S. et al. Shear stress regulates forward and reverse planar cell polarity of vascular endothelium in vivo and in vitro. Circ. Res. 98, 939-946 (2006).

6. Levesque, M. J. \& Nerem, R. M. The elongation and orientation of cultured endothelial cells in response to shear stress. J. Biomech. Eng. 107, 341-347 (1985).

7. Noria, S., Cowan, D. B., Gotlieb, A. I. \& Langille, B. L. Transient and steadystate effects of shear stress on endothelial cell adherens junctions. Circ. Res. 85, 504-514 (1999).

8. Seebach, J. et al. Regulation of endothelial barrier function during flow-induced conversion to an arterial phenotype. Cardiovasc. Res. 75, 596-607 (2007).

9. White, C. R. \& Frangos, J. A. The shear stress of it all: the cell membrane and mechanochemical transduction. Philos. Trans. Roy. Soc. Lond. B Biol. Sci. 362, 1459-1467 (2007).

10. Hahn, C. \& Schwartz, M. A. Mechanotransduction in vascular physiology and atherogenesis. Nat. Rev. Mol. Cell Biol. 10, 53-62 (2009).

11. Tarbell, J. M., Shi, Z.-D., Dunn, J. \& Jo, H. Fluid mechanics, arterial disease, and gene expression. Ann. Rev. Fluid Mech. 46, 591-614 (2014).

12. Bryan, M. T. et al. Mechanoresponsive networks controlling vascular inflammation. Arterioscler. Thromb. Vasc. Biol. 34, 2199-2205 (2014).

13. Traub, O. \& Berk, B. C. Laminar shear stress: mechanisms by which endothelial cells transduce an atheroprotective force. Arterioscler. Thromb. Vasc. Biol. 18, 677-685 (1998)

14. Davies, P. F., Civelek, M., Fang, Y. \& Fleming, I. The atherosusceptible endothelium: endothelial phenotypes in complex haemodynamic shear stress regions in vivo. Cardiovasc. Res. 99, 315-327 (2013).

15. Dai, G. et al. Distinct endothelial phenotypes evoked by arterial waveforms derived from atherosclerosis-susceptible and -resistant regions of human vasculature. Proc. Natl. Acad. Sci. USA 101, 14871-14876 (2004).

16. Chien, S. Effects of disturbed flow on endothelial cells. Ann. Biomed. Eng. 36, 554-562 (2008).

17. Malek, A. M., Alper, S. L. \& Izumo, S. Hemodynamic shear stress and its role in atherosclerosis. JAMA 282, 2035-2042 (1999).

18. Givens, C. \& Tzima, E. Endothelial mechanosignaling: does one sensor fit all? Antioxid. Redox Signal. 25, 373-388 (2016).

19. Garcia-Cardena, G. \& Slegtenhorst, B. R. Hemodynamic control of endothelial cell fates in development. Annu. Rev. Cell Dev. Bio. 32, 633-648 (2016).

20. Tarbell, J. M., Simon, S. I. \& Curry, F.-R. E. Mechanosensing at the vascular interface. Annu. Rev. Biomed. Eng. 16, 505-532 (2014).

21. Briot, A. et al. Endothelial NOTCH1 is suppressed by circulating lipids and antagonizes inflammation during atherosclerosis. J. Exp. Med. 212, 2147-2163 (2015).

22. Sivarapatna, A. et al. Arterial specification of endothelial cells derived from human induced pluripotent stem cells in a biomimetic flow bioreactor. Biomaterials 53, 621-633 (2015).

23. Theodoris, C. V. et al. Human disease modeling reveals integrated transcriptional and epigenetic mechanisms of NOTCH1 haploinsufficiency. Cell 160, 1072-1086 (2015).

24. Meloty-Kapella, L., Shergill, B., Kuon, J., Botvinick, E. \& Weinmaster, G. Notch ligand endocytosis generates mechanical pulling force dependent on dynamin, epsins and actin. Dev. Cell 22, 1299-1312 (2012).

25. Lawson, N. D. et al. Notch signaling is required for arterial-venous differentiation during embryonic vascular development. Development 128, 3675-3683 (2001)

26. Hasan, S. S. et al. Endothelial Notch signaling limits angiogenesis via control of artery formation. Nat. Cell Biol. 19, 928-940 (2017).

27. Pitulescu, M. E. et al. Dll4 and Notch signalling couples sprouting angiogenesis and artery formation. Nat. Cell Biol. 19, 915-927 (2017).

28. Villa, N. et al. Vascular expression of Notch pathway receptors and ligands is restricted to arterial vessels. Mech Dev. 108, 161-164 (2001).

29. Nowotschin, S., Xenopoulos, P., Schrode, N. \& Hadjantonakis, A.-K. A bright single-cell resolution live imaging reporter of Notch signaling in the mouse. BMC Dev. Biol. 13, 15 (2013).

30. Tzima, E. et al. A mechanosensory complex that mediates the endothelial cell response to fluid shear stress. Nature 437, 426-431 (2005).

31. Mack, J. J. et al. Real-time maps of fluid flow fields in porous biomaterials. Biomaterials 34, 1980-1986 (2013).

32. Shirali, A. S., McDonald, A. I., Mack, J. J. \& Iruela-Arispe, M. L. Reproducible arterial denudation injury by infrarenal abdominal aortic clamping in a murine model. J. Vis. Exp. 117, e54755 (2016)

33. Wang, C., Baker, B. M., Chen, C. S. \& Schwartz, M. A. Endothelial cell sensing of flow direction. Arterioscler. Thromb. Vasc. Biol. 33, 2130-2136 (2013).

34. Coan, D. E., Wechezak, A. R., Viggers, R. F. \& Sauvage, L. R. Effect of shear stress upon localization of the Golgi apparatus and microtubule organizing center in isolated cultured endothelial cells. J. Cell Sci. 104, 1145-1153 (1993).

35. McManus, K. J. \& Hendzel, M. J. ATM-dependent DNA damage-independent mitotic phosphorylation of $\mathrm{H} 2 \mathrm{AX}$ in normally growing mammalian cells. $\mathrm{Mol}$. Biol. Cell 16, 5013-5025 (2005).

36. Chapin, J. C. \& Hajjar, K. A. Fibrinolysis and the control of blood coagulation. Blood Rev. 29, 17-24 (2015).

37. Hogan, P. G., Chen, L., Nardone, J. \& Rao, A. Transcriptional regulation by calcium, calcineurin, and NFAT. Genes Dev. 17, 2205-2232 (2003).

38. Kumar, S. et al. Accelerated atherosclerosis development in C57Bl6 mice by overexpressing AAV-mediated PCSK9 and partial carotid ligation. Lab Invest 97, 935-945 (2017).

39. Miao, H. et al. Effects of flow patterns on the localization and expression of VEcadherin at vascular endothelial cell junctions: in vivo and in vitro investigations. J. Vasc. Res. 42, 77-89 (2005).

40. Dekker, R. J. et al. Prolonged fluid shear stress induces a distinct set of endothelial cell genes, most specifically lung Krüppel-like factor (KLF2). Blood 100, 1689-1698 (2002).

41. Nayak, L., Lin, Z. \& Jain, M. K. Go with the flow: how Krüppel-like factor 2 regulates the vasoprotective effects of shear stress. Antiox. Redox Sig. 15, 1449-1461 (2011).

42. Paszkowiak, J. J. \& Dardik, A. Arterial wall shear stress: observations from the bench to the bedside. Vasc. Endovasc. Surg. 37, 47-57 (2003).

43. Wasserman, S. M. \& Topper, J. N. Adaptation of the endothelium to fluid flow: in vitro analyses of gene expression and in vivo implications. Vasc. Med. 9 , 35-45 (2004)

44. Hellstrom, M. et al. Dll4 signalling through Notch1 regulates formation of tip cells during angiogenesis. Nature 445, 776-780 (2007).

45. Bentley, K. et al. The role of differential VE-cadherin dynamics in cell rearrangement during angiogenesis. Nat. Cell. Biol. 16, 309-321 (2014).

46. Lawson, N. D. et al. Notch signaling is required for arterial-venous differentiation during embryonic vascular development. Development 128, 3675-3683 (2001).

47. Choi, D. et al. Laminar flow downregulates Notch activity to promote lymphatic sprouting. J. Clin. Invest. 127, 1225-1240 (2017).

48. Jahnsen, E. D. et al. Notch1 is pan-endothelial at the onset of flow and regulated by flow. PLoS ONE 10, e0122622 (2015).

49. Noseda, M. et al. Notch activation induces endothelial cell cycle arrest and participates in contact inhibition: role of p21Cip1 repression. Mol. Cell. Biol. 24, 8813-8822 (2004).

50. Sainson, R. C. et al. Cell-autonomous notch signaling regulates endothelial cell branching and proliferation during vascular tubulogenesis. FASEB J. 19, 1027-1029 (2005).

51. Wheeler, D. G., Barrett, C. F., Groth, R. D., Safa, P. \& Tsien, R. W. CaMKII locally encodes L-type channel activity to signal to nuclear CREB in excitationtranscription coupling. J. Cell Biol. 183, 849-863 (2008).

52. Wang, C. et al. Apelin protects sarcoplasmic reticulum function and cardiac performance in ischemia-reperfusion by attenuating oxidation of sarcoplasmic reticulum $\mathrm{Ca}^{2+}$-ATPase and ryanodine receptor. Cardiovasc. Res. 100, 114-124 (2013).

53. McClain, J. L. \& Gulbransen, B. D. The acute inhibition of enteric glial metabolism with fluoroacetate alters calcium signaling, hemichannel function, and the expression of key proteins. J. Neurophysiol. 117, 365-375 (2017).

54. Clapham, D. E. Calcium signaling. Cell 131, 1047-1058 (2007)

55. Humeau, J. et al. Calcium signaling and cell cycle: progression or death. Cell Calcium (in the press).

56. Skelding, K. A., Rostas, J. A. \& Verrills, N. M. Controlling the cell cycle: the role of calcium/calmodulin-stimulated protein kinases I and II. Cell Cycle 10, 631-639 (2011).

57. Brown, R. C. \& Davis, T. P. Calcium modulation of adherens and tight junction function: a potential mechanism for blood-brain barrier disruption after stroke. Stroke 33, 1706-1711 (2002).

58. Gasperini, R. J. et al. How does calcium interact with the cytoskeleton to regulate growth cone motility during axon pathfinding? Mol. Cell Neurosci. (in the press).

59. Bhatt, A., Kaverina, I., Otey, C. \& Huttenlocher, A. Regulation of focal complex composition and disassembly by the calcium-dependent protease calpain. J. Cell Sci. 115, 3415-3425 (2002)

60. Shen, J., Luscinskas, F. W., Connolly, A., Dewey, C. F. \& Gimbrone, M. A. Fluid shear stress modulates cytosolic free calcium in vascular endothelial cells. Am. J. Physiol. 262, C384-C390 (1992).

61. Liu, B., Lu, S., Zheng, S., Jiang, Z. \& Wang, Y. Two distinct phases of calcium signalling under flow. Cardiovasc. Res. 91, 124-133 (2011).

62. Helmlinger, G., Berk, B. C. \& Nerem, R. M. Calcium responses of endothelia cell monolayers subjected to pulsatile and steady laminar flow differ. Am. J. Physiol. 269, C367-C375 (1995).

63. Yokota, Y. et al. Endothelial $\mathrm{Ca}^{2+}$ oscillations reflect VEGFR signalingregulated angiogenic capacity in vivo. eLife 4, e08817 (2015). 
64. Blackman, B. R., Garcia-Cardena, G. \& Gimbrone, M. A. A new in vitro model to evaluate differential responses of endothelial cells to simulated arterial shear stress waveforms. J. Biomech. Eng. 124, 397-407 (2002).

65. Youssef, K., Mack, J. J., Iruela-Arispe, M. L. \& Bouchard, L.-S. Macro-scale topology optimization for controlling internal shear stress in a porous scaffold bioreactor. Biotechnol. Bioeng. 109, 1844-1854 (2012).

66. Callaghan, P. Principles of Nuclear Magnetic Resonance Microscopy, Vol. 3 (Clarendon, Oxford, UK, 1991).

67. Bacabac, R. G. et al. Dynamic shear stress in parallel-plate flow chambers. $J$. Biomech. 38, 159-167 (2005).

68. Grynkiewicz, G., Poenie, M. \& Tsien, R. Y. A new generation of $\mathrm{Ca}^{2+}$ indicators with greatly improved fluorescence properties. J. Biol. Chem. 260, 3440 (1985).

69. Briot, A. et al. Repression of Sox 9 by Jag1 is continuously required to suppress the default chondrogenic fate of vascular smooth muscle cells. Dev. Cell 31, 707-721 (2014).

70. Lizama, C. O. et al. Repression of arterial genes in hemogenic endothelium is sufficient for haematopoietic fate acquisition. Nat. Commun. 6, 7739 (2015).

\section{Acknowledgments}

This work was supported by funds from the Ruth L. Kirschstein National Research Service Award (T32 HL069766) (to J.J.M. and B.J.A.) and grants from the National Institutes of Health (1 R01 HL114086-01A) (to M.L.I.A. and L.S.B.), (1RO1HL130290) (to M.L.I.A.), (P01HL030568) (to A.M.F. and M.L.I.A.), and (R00 HL103789) (to Y.F.). T.S.M. and T.S. acknowledge support from a QCB Collaboratory Postdoctoral Fellowship and the QCB Collaboratory community directed by Dr. Matteo Pellegrini. We thank Dr. Hanjoong Jo for the generous gift of PCSK9 AAV and control AAV used in the atherosclerosis study. We are grateful to Dr. Georg Hilfenhaus for performing tail vein injections, Dr. Aditya Shirali for executing the arterial denudation surgery, Lauren Serrano and Alexander Iruela Lane for aorta imaging and analysis of atherosclerosis, Joan Cheng and Dr. Bethan Clifford for assistance with retro-orbital bleeds and lipid analysis, and Michelle Steel for maintaining the mouse colonies used in this study. We also acknowledge the contribution of the Center for Systems Biomedicine and UCLA Vector Core for the NanoString analysis.

\section{Author contributions}

J.J.M. and M.L.I.A designed the project. J.J.M., W.M.J., H.S., A.B. and R.H.-S. performed in vitro experiments with the aid of C.-H.K., Y.F. and T.F.L.; J.J.M and M.L.I.A. designed and performed in vivo experiments with the aid of E.J.T., T.Q.d.A.V., C.O.L. and A.C.Z B.J.A. and L.S.B. designed and performed the MRI flow mapping analyses. T.S.M. and G.C.F. performed analyses of calcium imaging and T.S.M. quantified protein polarization kinetics. A.I.M. contributed to confocal imaging and T.S. and M.C.R. aided in microarray analysis. E.J.T., T.Q.d.A.V., M.N. and A.M.F. contributed to study design and data interpretation. J.J.M. and M.L.I.A. wrote the manuscript with the contribution of all the authors.

\section{Additional information}

Supplementary Information accompanies this paper at doi:10.1038/s41467-017-01741-8.

Competing interests: A.M.F. and M.N. are principals in Bruin Pharma and A.M.F. is an officer in Bruin Pharma. The remaining authors declare no competing financial interests.

Reprints and permission information is available online at http://npg.nature.com/ reprintsandpermissions/

Publisher's note: Springer Nature remains neutral with regard to jurisdictional claims in published maps and institutional affiliations.

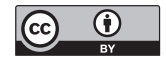

Open Access This article is licensed under a Creative Commons Attribution 4.0 International License, which permits use, sharing, adaptation, distribution and reproduction in any medium or format, as long as you give appropriate credit to the original author(s) and the source, provide a link to the Creative Commons license, and indicate if changes were made. The images or other third party material in this article are included in the article's Creative Commons license, unless indicated otherwise in a credit line to the material. If material is not included in the article's Creative Commons license and your intended use is not permitted by statutory regulation or exceeds the permitted use, you will need to obtain permission directly from the copyright holder. To view a copy of this license, visit http://creativecommons.org/ licenses/by/4.0/

(c) The Author(s) 2017 